\title{
Optimal Bidding Strategies for Thermal and Generic Programming Units in the Day-Ahead Electricity Market
}

\author{
F.-Javier Heredia, Marcos J. Rider, Member, IEEE, and Cristina Corchero
}

\begin{abstract}
This study has developed a stochastic programming model that integrates the day-ahead optimal bidding problem with the most recent regulation rules of the Iberian Electricity Market (MIBEL) for bilateral contracts (BC), with a special consideration for the new mechanism to balance the competition of the production market, namely virtual power plant (VPP) auctions. The model allows a price-taking generation company (GenCo) to decide on the unit commitment of the thermal units, the economic dispatch of the BCs between the thermal units and the generic programming unit (GPU), and the optimal sale/purchase bids for all units (thermal and generic), by observing the MIBEL regulation. The uncertainty of the spot prices has been represented through scenario sets built from the most recent real data using scenario reduction techniques. The model has been solved using real data from a Spanish generation company and spot prices, and the results have been reported and analyzed.
\end{abstract}

Index Terms-Bilateral contracts, electricity spot market, optimal bidding strategies, short-term electricity generation planning, stochastic programming, virtual power plant auctions.

\section{NOTATION}

The following are the notations used throughout this paper. Sets:

$\mathcal{I} \quad$ Set of hourly intervals.

$\mathcal{S} \quad$ Set of scenarios.

$\mathcal{B} \quad$ Set of bilateral contracts.

$\mathcal{T} \quad$ Set of thermal generation units.

$\mathcal{M}_{i}^{S} \quad$ Set of scenarios with conditioned accepted GPU's sale bid.

$\mathcal{M}_{i}^{P} \quad$ Set of scenarios with conditioned accepted GPU's purchase bid.

$\mathcal{U}^{T} \quad$ Set of initial condition of unit commitment binary variables.

Manuscript received November 17, 2008; revised March 28, 2009. First published February 08, 2010; current version published July 21, 2010. This work was supported by the Ministry of Science and Technology of Spain through CICYT Project DPI2005-09117-C02-01 and MICINN Project DPI2008-02153. Paper no. TPWRS-00930-2008.

The authors are with the Statistics and Operations Research Department, Universitat Politècnica de Catalunya, 08034 Barcelona, Spain (e-mail: f.javier.heredia@upc.edu; marcos.rider@upc.edu; cristina.corchero@upc.edu). Digital Object Identifier 10.1109/TPWRS.2009.2038269
$\mathrm{K}_{i t}^{T, s} \quad$ Feasible set of the equivalent thermal-matched energy constraints.

$\mathrm{K}_{i}^{S} \quad$ Feasible set of the equivalent generic-matched sale energy constraints.

$\mathrm{K}_{i}^{P} \quad$ Feasible set of the equivalent generic-matched purchase energy constraints.

$\mathrm{K}_{i}^{R} \quad$ Feasible set of the equivalent generic-matched residual energy constraints.

Constants:

$P^{s} \quad$ Probability of scenario $s$.

$c_{t}^{b} \quad$ Base procurement cost of unit $t(€)$.

$c_{t}^{l} \quad$ Linear procurement cost of unit $t(\Theta / M W h)$.

$c_{t}^{q} \quad$ Quadratic procurement cost of unit $t\left(\Theta / \mathrm{MWh}^{2}\right)$.

$p_{i t}^{D, s} \quad$ Bilateral-free day-ahead matched energy, unit $t$, hour $i$, scenario $s$ (MWh).

$L_{i j}^{B} \quad$ Energy of the BC $j$ in hour $i(\mathrm{MWh})$.

$\lambda_{i j}^{B}$

$\lambda^{S}$

Unit profit of the $\mathrm{BC} j$ in hour $i(\epsilon / \mathrm{MWh})$.

Unit profit of the sale $\mathrm{BC}$ after the day-ahead market ( $\Theta / M W h)$.

$\bar{b}^{S} \quad$ Maximum energy that can be sold through the BC after the day-ahead market (MWh).

$\lambda^{P} \quad$ Unit cost of the purchase $\mathrm{BC}$ after the day-ahead market ( $\mathrm{C} / \mathrm{MWh}$ ).

$\bar{b}^{P} \quad$ Maximum energy that can be purchased through the $\mathrm{BC}$ after the day-ahead market (MWh).

$\lambda^{V} \quad$ Virtual power plant exercise price $(\mathbb{E} / \mathrm{MWh})$.

$\bar{p}^{V} \quad$ Capacity of the virtual power plant (MWh).

$\bar{p}_{t} \quad$ Maximum generation of unit $t$ (MW).

$\underline{p}_{t} \quad$ Minimum generation of unit $t(\mathrm{MW})$.

$c_{t}^{o n} \quad$ Start-up cost of unit $t(€)$.

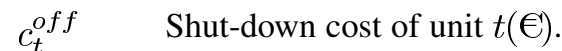

$s t_{t}^{0} \quad$ Initial state of unit $t$ (hours).

$t_{t}^{o n}$
Operational minimum in service time of unit $t$ (hours). 
$t_{t}^{o f f} \quad$ Operational minimum idle time of unit $t$ (hours).

$\lambda_{i}^{D, s} \quad$ Day-ahead (spot) market price in hour $i$, scenario $s(\Theta / M W h)$.

Functions:

$\lambda_{i t}^{*} \quad$ Optimal sale bid function of unit $t$ in hour $i(\mathrm{E} / \mathrm{MWh})$.

$B^{T, s} \quad$ Bilateral-free benefit function

First-stage continuous variables:

$b_{i t j}^{T} \quad$ Generation of unit $t$ in hour $i$ allocated to the $\mathrm{BC} j(\mathrm{MWh})$.

$p_{i}^{V} \quad$ Virtual power plant capacity used in hour $i$ (MWh).

$b_{i j}^{G} \quad$ Generic programming unit's generation in hour $i$ allocated to the $\mathrm{BC} j(\mathrm{MWh})$.

$w_{i}^{S} \quad$ Auxiliary variable used in the definition of the sale-matched energy of the GPU.

$w_{i}^{P} \quad$ Auxiliary variable used in the definition of the purchase-matched energy of the GPU.

$w_{i}^{R} \quad$ Auxiliary variable used in the definition of the residual purchase-matched energy of the GPU.

First-Stage Binary Variables:

$x_{i}^{V} \quad$ Equal to 1 if the VPP rights are exercised, 0 otherwise.

$u_{i t} \quad$ Equal to 1 if the thermal unit $t$ must be committed in hour $i, 0$ otherwise.

$a_{i t} \quad$ Equal to 1 if the thermal unit $t$ must be turned-on in hour $i, 0$ otherwise.

$e_{i t} \quad$ Equal to 1 if the thermal unit $t$ must be shut-down in hour $i, 0$ otherwise.

$y_{i}^{S} \quad$ Auxiliary variable used in the definition of the sale-matched energy of the GPU.

$y_{i}^{P} \quad$ Auxiliary variable used in the definition of the purchase-matched energy of the GPU.

$y_{i}^{R} \quad$ Auxiliary variable used in the definition of the residual purchase-matched energy of the GPU.

Second-Stage Continuous Variables:

$v_{i t}^{s} \quad$ Auxiliary variables used in the definition of the matched energy of the thermal units at period $i$, scenario $s$.

$b_{i}^{S, s} \quad$ Sale BC after the day-ahead market in hour $i$ and scenario $s(\mathrm{MWh})$.

$b_{i}^{P, s} \quad$ Purchase BC after the day-ahead market in hour $i$ and scenario $s$ (MWh).

$p_{i t}^{s} \quad$ Total thermal generation of unit $t$ in hour $i$, scenario $s$ (MWh).

$$
\begin{aligned}
& p_{i t}^{T, s} \\
& p_{i}^{S, s}
\end{aligned}
$$$$
p_{i}^{P, s}
$$$$
p_{i}^{R, s}
$$

\section{Second-Stage Binary Variables:}

Auxiliary variables used in the definition of the matched energy of the thermal units in hour $i$, scenario $s$.

\section{INTRODUCTION}

$\mathbf{T}$ HE new rules of the electrical energy production market operation of the Iberian Electricity Market MIBEL (mainland Spanish and Portuguese systems), for the daily and intradaily market from June 2007 [1], have introduced mechanisms to encourage competition in the production market (physical futures contracts, bilateral contracts (BCs) and virtual power plant (VPP) capacity), and have brought new challenges to the modeling and optimization of the market operation.

To increase the proportion of electricity that is purchased through BCs with a duration of several months and to stimulate liquidity in forward electricity markets, the Royal Decree 1634/2006, dated December 29, 2006 [2], has imposed the requirement of holding a series of five auctions offering VPP capacity to any party that is a member of the MIBEL, on Endesa and Iberdrola (the two dominant utility companies in the Spanish electricity market). In 2006, the total installed capacity of both the companies was around $47 \mathrm{GW}$, and the total installed capacity of the Spanish electricity system was $78.3 \mathrm{GW}$. Fig. 1 shows the volumes to be auctioned by Endesa and Iberdrola with respect to the Royal Decree 1634/2006. It can be observed that the greatest volumes of auctioned VPP capacity were reached from April to September 2008, with a total amount of $2000 \mathrm{MW}$ [3].

Further experience of the application of VPP auctions can be found in France, where the Electricité de France (EDF), since September 2001, has made available 5.4 GW of generation capacity in France to facilitate the liberalization of the French electricity market [4]. On July 4, 2003, the Belgian Competition Council approved various transactions leading to the appointment of Electrabel Customer Solutions, a subsidiary of Electrabel, as the default supplier for the customers of several intermunicipal distribution companies, subject to certain undertakings. As a part of these undertakings, Electrabel has agreed to offer up to a maximum of $1.2 \mathrm{GW}$ of VPP capacity in Belgium to the actual or potential competitors [5]. On September 19, 2007, E.ON Sales \& Trading GmbH (EST) offered 250 MW of the VPP product in a first auction to the electricity market in Germany. EST is believed to consider conducting further auctions for one or more similar products on an annual basis [6].

In Spain, the VPP capacity indicates that the buyer of this product will have the capacity to generate MWh at his disposal. The buyer can exercise the right to produce against an exercise 


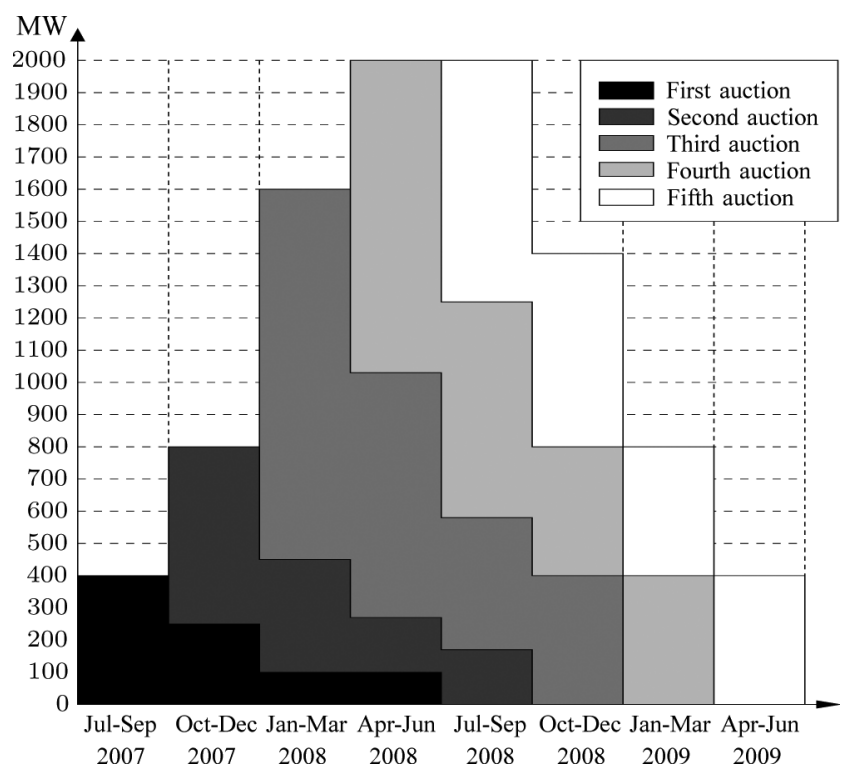

Fig. 1. Five auctions of the VPP capacity of the Spanish peninsular electricity market.

price, set in advance, by paying an option premium. Hence, although Endesa and Iberdrola still own the power plants, part of their production capacity will be at the disposal of the buyers of VPP, who are the subjects of our study. There will be base-load and peak-load contracts with different strike prices that are defined a month before the auction. In each case, contracts with the duration of three, six, and twelve months will be offered. Furthermore, all the products will be offered simultaneously using an electronic auction. Fig. 2 shows the evolution of the day-ahead market price $\lambda_{i}^{D}$ of day $i$ (daily maximum, mean, and minimum) from July 2007 to April 2008, and the four first pre-defined strike price for base- and peak-load VPP capacity, respectively. The energy resulting from the exercise of the VPP options can be used by the buyers both to contribute to cover the national and international BCs prior to the day-ahead market as well as to sell it to the day-ahead market. In this latter case, the unmatched VPP energy, if any, can be sold through national BCs after the day-ahead market. These new BCs after the day-ahead market are negotiated between the agents prior to day-ahead market gate closure, and must not be confused with other subsequent markets such as the reserve or balancing markets (see [1] and [7]).

\section{A. Literature Review}

The VPP capacity auctions attempt to reduce the influence of the dominant agents through financial tools to increase the competition in the market. This kind of regulation aims to move towards a perfectly competitive market which is achieved by all price-taking operators. Because of those reasons and the fact that it is very difficult to model the influence of a price-maker operator in the clearing price, the majority of the publications are focused on price-taking generation companies. The general considerations about the bidding process in these electricity markets can be found in [8]-[10].

Several authors have proposed optimal bidding models in the day-ahead market for thermal units under the price-taker assumption, with or without BCs. The authors in [11] presented a

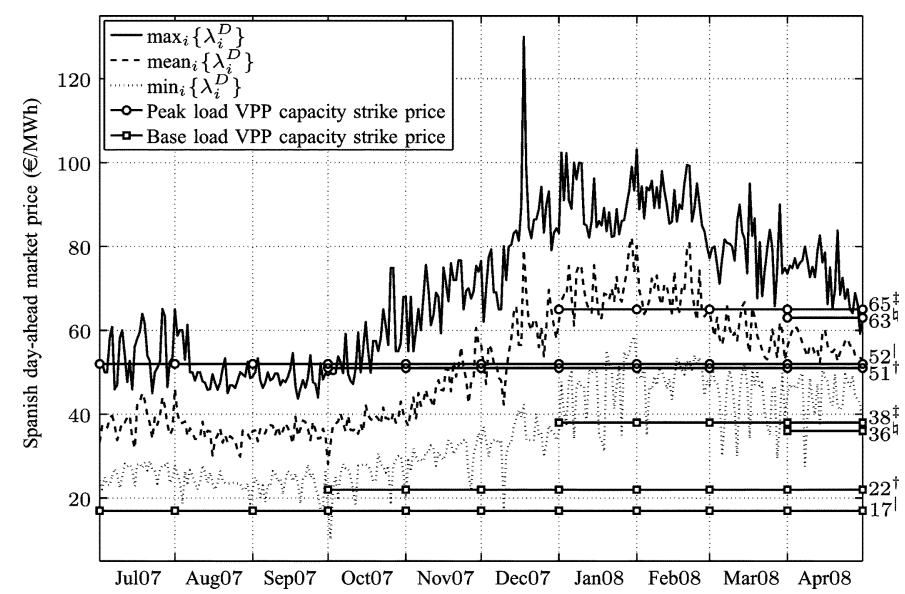

Fig. 2. Spanish day-ahead price market and strike price for the base- and peakload VPP capacity ('First auction, † Second auction, $\ddagger$ Third auction, kFourth auction).

mixed integer programming model to optimize the production scheduling of a single unit with a simple bidding strategy. The approximation of the step-wise bidding curves by linear functions based on the marginal costs was already considered in [12] and [13], although in a context without BCs. In [14], the concept of price-power function, which is similar to the matched energy function defined in this paper, was used to derive the optimal offer curves of a hydrothermal system under the assumption that the spot prices for the day-ahead and reserve markets behave as a Markov chain. The mixed-integer stochastic programming model presented in [15] distinguishes between the variables corresponding to the bid energy and those representing the matched energy, although in a price-maker framework and without BCs. An earlier model [16] is found to be closely related in some aspects to the one presented in this study, where a stochastic unit commitment problem with BC is solved by maximizing the day-ahead market benefit. Stochasticity in the spot prices is introduced through a set of scenarios, giving rise to a two-stage stochastic programming problem. In [17], the authors presented a mixed-integer stochastic optimization model for scheduling thermal units, where the production plans were optimized in the presence of stochastic market clearing prices. Nevertheless, the models in [16] and [17] did not propose any explicit modeling of the optimal bidding. To our knowledge, there are no publications that consider either the BCs after the day-ahead market or the modeling of the VPP.

Regarding the risk in the short-term horizon, there are authors who have included it in the optimization models [14] and other studies that have not considered introducing risk [16]. Usually, the latter approaches consider risk as something that must be taken into account in the mid-term horizon jointly with other mid-term strategies, such as fuel or derivatives contracts [18]. In this work, we have focused on the optimal bidding model corresponding to a given $\mathrm{BC}$ portfolio; thus, we consider that risk has been hedged when developing this contract portfolio.

\section{B. Contributions}

This study has developed a stochastic mixed-integer quadratic programming model for a price-taking generation company (GenCo) that has acquired VPP capacity options. 
The energy of the VPP options is integrated into the MIBEL's energy production system through the so-called generic programming unit (GPU) ${ }^{1}$ which will be described in the next section. The objective of this work has been to find the optimal bidding strategy of both the thermal production units and the GPU in the Spanish day-ahead electricity market under the most recent MIBEL regulation regarding the BCs rules. The model allows a price-taking generation company to decide about the unit commitment of its thermal units, the economic dispatch of the BCs between the thermal and generic units, and the optimal bid for both thermal and generic units, based on the MIBEL regulation. The model has been tested with the real data from a Spanish generation company and spot-market prices. It has been implemented with AMPL and solved with CPLEX.

The main contributions of this paper are as follows:

- a new model for the optimal thermal bid function and matched energy which takes into account the presence of BCs;

- the mathematical modeling of the GPU and the VPP;

- the modeling of the optimal bid functions and matched energy of the GPU;

- the inclusion in the optimization model of the BCs after the day-ahead market;

- the consideration of the most recent regulations of the MIBEL energy market.

The GPU and VPP are the new elements in the MIBEL, whose utilities need to be integrated into their daily optimal bid policy. These elements have not been considered previously in the literature. The model presented is the first attempt both to use and analyze these novel elements aiming to encourage the competition in the MIBEL, and can be of great economic interest for any GenCo operating a GPU. Regarding the VPP, the model provides the GenCo with a tool to decide if the energy rights of the VPP should be nominated or not. Regarding the GPU, the model's output determines its optimal bid to the market and the participation in the BCs. The numerical experiments presented in Section V show that this optimal bid policy can increase the day-ahead market benefits of a GenCo operating a GPU at $10 \%$ or even more for a GenCo holding the VPP capacity.

Finally, it must be mentioned that another relevant novelty of the paper is the consideration, for the first time to our knowledge, of the BCs after the day-ahead market, which is another characteristic of the MIBEL.

This paper is organized as follows. Section II describes the MIBEL's energy production system around the day-ahead market and the relevance of the GPU. In Section III, the stochastic programming model for the optimal bidding strategy is developed. In Section IV, the market price scenario generation procedure is described. In Section V, a detailed case study is presented and solved with the proposed stochastic programming model, reporting and analyzing the computational results. Finally, some relevant conclusions are drawn in Section VI.

\section{MIBEL'S ENERGY PRODUCTION SYSTEM}

The day-ahead market is not only the main physical energy market of Portugal and Spain in terms of the amount of traded

\footnotetext{
${ }^{1}$ Translation of "unidad de programación genérica", the official Spanish term used in the MIBEL's documentation [1].
}

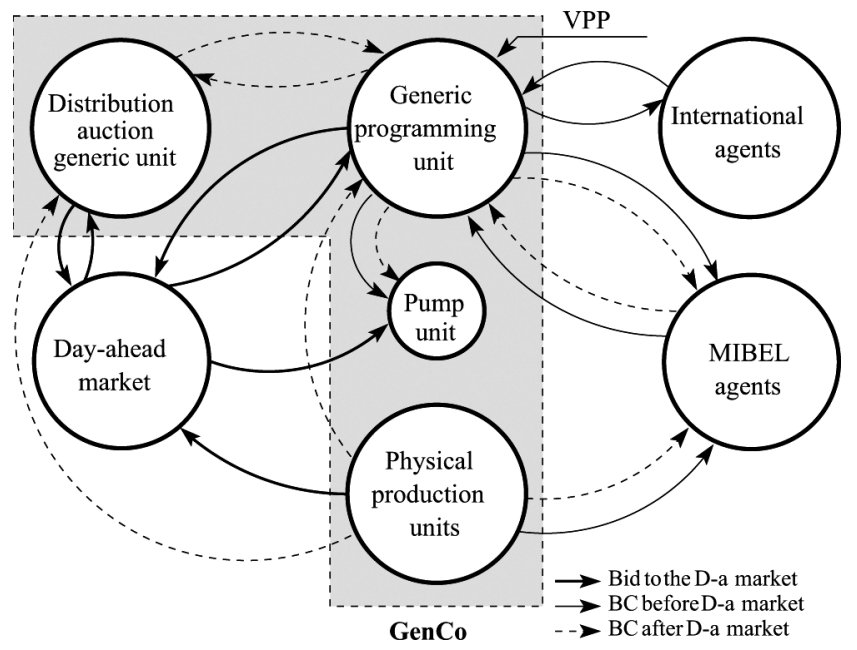

Fig. 3. GenCo's operation problem on the MIBEL's energy production system around the day-ahead (D-a) market.

energy, but also the mechanism through which other energy products, such as BCs, are integrated into the MIBEL's energy production system. Fig. 3 depicts the elements of the MIBEL's energy production system relevant to the decision-making problem of a GenCo that owns the VPP options. These elements are:

- The programming units owned by the GenCo (nodes in the dashed area). These units could be either physical (thermal, nuclear, combined-cycle, hydro, pump units) or generic, i.e., virtual units through which the GenCo can operate by either selling or buying energy, by both bidding to the pool and settling BC with the other participant in the MIBEL's energy production system.

- The pool (day-ahead market node) to which all the programming units of the GenCo can submit sale bids (physical production units), purchase bids (pump units), or both sale/purchase bids (the generic units). The allowed bids are represented in Fig. 3 by the thick arcs.

- There are two agents that interact with the GenCo's programming units: the International agents and the MIBEL agents. The GenCo can buy/sell energy from/to the surrounding foreign generation areas-namely, Portugal, Morocco, and France-through the international BCs settled with its GPU. There are also BCs signed both by the GPU and the physical production units with the rest of the MIBEL agents. These BCs can be of two kinds: before the day-ahead market (continuous arcs in Fig. 3) and after the day-ahead market (dashed arcs). The characteristics of these BCs are explained in Section III-A.

\section{A. Bids to the Day-Ahead Market}

The thick arcs in Fig. 3 correspond to the possible sale/purchase bids submitted to the day-ahead market of day D. In the MIBEL, this market consists of a series of 24-h auctions that are cleared simultaneously between 10:00 and 10:30 $\mathrm{h}$ of the previous day (D-1). Selling and buying agents must submit their sale/purchase bids to each auction before 10:00 $\mathrm{h}$ of D-1. Both sale and purchase bids are composed of up to 25 price-energy pairs with nonincreasing price values and each agent is unaware 
of the bids of the rest of the agents. The thermal production units engaged in dispatching the BCs are allowed to offer the nondispatched part of its total capacity to the pool. The clearing price $\lambda_{i}^{s}$ of each hourly auction $i$ is determined by the intersection of the aggregated offer and demand curves. All the sale/purchase bids with a lower/greater bid price are matched and will be remunerated at the same clearing price $\lambda_{i}^{s}$ irrespective of the original bid price.

\section{B. Bilateral Contracts}

BCs are agreements to provide a given amount of electrical energy at a stipulated price along with a delivery period. The characteristics of the $\mathrm{BC}$ (energy, price and delivering period) are negotiated among the MIBEL agents before the day-ahead market, either in organized or nonorganized markets. There are two organized BC markets in the MIBEL, the CESUR and VPP auctions ([19] and [3], respectively). On day D-3, the CESUR auction settles the BCs signed by generation and distribution companies to supply power at regulated prices in the peninsula. The distribution auction generic unit (see Fig. 3) is used by the GenCo to integrate the energy matched by the CESUR auction into the energy production system. By law, a GenCo holding such a BC must use this generic unit to submit a purchase bid at the maximum technical price to the day-ahead market for the entire amount of the contract, and therefore, there is no room for optimization. The VPP auctions have already been described in Section I. The exercised energy of the VPP is integrated into the energy production system through the GPU. This GPU brings more flexibility to the GenCo operations in the MIBEL, because with the GPU, the utility can:

- integrate the VPP exercised energy into the energy production system, both offering this energy to the pool through sales bids and allocating it among the GenCo's portfolio of national and international $\mathrm{BCs}$;

- act as a purchase agent, both sending purchase bids to the pool and acquiring energy through national and international BCs.

In nonorganized $\mathrm{BC}$ markets, the producers and consumers agree on the amount, price, and period of the energy delivered. This agreement is set during a private negotiation. For a GenCo, these BCs usually represent a scheduled load curve, chargeable at a fixed price that has to be optimally dispatched among the GenCo's units. In the case of BCs before the day-ahead market, the resulting dispatch must be communicated (nominated using the MIBEL's terminology) to the system and market operator no later than $25 \mathrm{~min}$ before the closure of the day-ahead market (thin solid lines in Fig. 3). For the BCs after the day-ahead market, the dispatch must be nominated within 30 min following the publication of the day-ahead market clearing results (dashed lines in Fig. 3). After market clearing, the generation program of the GPU must be allocated among the GenCo's physical production units and $\mathrm{BCs}$, in such a way that the net energy balance of the GPU is zero. The existence of BCs after the day-ahead market prevents violation of the aforementioned netting energy balance condition as a consequence of possible unmatched GPU's sale or purchase bids.

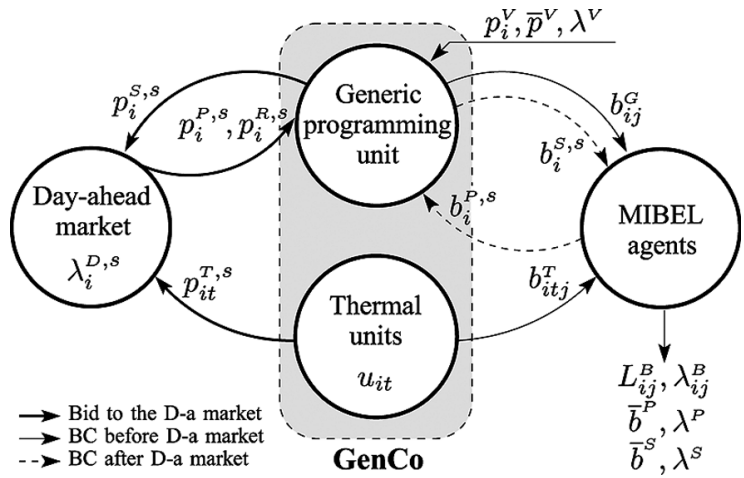

Fig. 4. Case study.

\section{Stochastic Programming Model}

Fig. 4 represents the part of the whole MIBEL energy production system (Fig. 3) considered in this study. This system will be modeled based on the following assumptions:

- The GenCo is a price-taker operating in the MIBEL with a set $\mathcal{T}$ of thermal units (coal, nuclear, gas, oil) and a GPU.

- The thermal units in $\mathcal{T}$ have linear or convex quadratic generation cost function, constant start-up/shut-down costs, and minimum generation/down time.

- The GPU is associated with a VPP with known capacity $\left(\bar{p}^{V} \mathrm{MWh}\right)$ and exercise price $\left(\lambda^{V} \mathrm{\Theta} / \mathrm{MWh}\right)$.

- Both thermal units and GPU bid to the $i \in \mathcal{I}=$ $\{1,2, \ldots, 24\}$ hourly auctions of the day-ahead market. The stochasticity of the spot price $\lambda_{i}^{D}, i \in \mathcal{I}$ is represented by a set of $\mathcal{S}$ scenarios.

- There is a portfolio $\mathcal{B}$ of BCs duties before the day-ahead market with the rest of the MIBEL agents, with known energy $\left(L_{i j}^{B} \mathrm{MWh}\right)$ and price $\left(\lambda_{i j}^{B} \in / \mathrm{MWh}\right)$.

- There is an agreement for selling (purchasing) BCs after the day-ahead market up to a quantity $\bar{b}^{S}$ MWh $\left(\bar{b}^{P}\right.$ MWh $)$ at a price $\lambda^{S} \in / \mathrm{MWh}\left(\lambda^{P} \in / \mathrm{MWh}\right)$. We assume that it is not possible to obtain net gain from those contracts $\left(\lambda^{P}>\lambda^{S}\right)$.

The objective of this study is to determine how to optimally manage the thermal units $\mathcal{T}$ and the GPU, to obtain the maximum benefit from the day-ahead market while covering the BCs agreements. This problem has been modeled in this work as a mixed integer quadratic two-stage stochastic optimization problem. Among the complete list of the variables of this model (see the Notation section), the main information provided by the model (here and now decisions or first-stage variables) for each period $i \in \mathcal{I}$ are as follows:

- For each thermal unit $t \in \mathcal{T}$, the unit commitment $\left(u_{i t}\right)$, the energy allocated to each BC, $b_{i t j}^{T}, \forall j \in \mathcal{B}$ and the optimal sale bids, expressed as a function of $b_{i t j}^{T}$ (see Section III-C).

- For the GPU, the exercised VPP energy $\left(p_{i}^{V}\right)$, the energy allocated to the BCs before the day-ahead market $\left(b_{i j}^{G}, \forall j \in \mathcal{B}\right)$, and the optimal sale/purchase bids, expressed in terms of $b_{i j}^{G}$ and $p_{i}^{V}$ (see Section III-D).

It must be noted that following the explanation given in Section II, all these variables represent quantities that the 
GenCo has to decide on before the day-ahead market clearing, and thus, must be associated with the first-stage variables.

The model presented in this study has been tuned to incorporate the specificities of the MIBEL energy production system, namely, the BCs after the day-ahead market, the integration of the nominated bilateral contracts into the sale thermal bid, and the capability of the GPU to submit purchase bids. Nevertheless, apart from the aforementioned characteristics, the proposed model could also be of interest for other electricity markets with VPP, such as the Belgian and German markets.

\section{A. Bilateral Contracts Constraints}

The GenCo has agreed to physically provide the energy amounts $L_{i j}^{B}$ at hour $i \in \mathcal{I}$ of day $\mathrm{D}$ for each one of the $j \in \mathcal{B}$ BCs with the rest of the MIBEL participants. This energy $L_{i j}^{B}$ can be provided both by the real thermal units $\mathcal{T}$ and the virtual GPU

$$
\left.\begin{array}{c}
\sum_{t \in \mathcal{T}} b_{i t j}^{T}+b_{i j}^{G}=L_{i j}^{B} \\
b_{i t j}^{T} \geq 0 \quad \forall t \in \mathcal{T} \\
b_{i j}^{G} \geq 0
\end{array}\right\} \begin{aligned}
& \forall i \in \mathcal{I} \\
& \forall j \in \mathcal{B} .
\end{aligned}
$$

\section{B. Thermal Unit Commitment}

Following [20], (2) can be used to formulate the minimum up and down times for thermal unit $t$

$$
\begin{gathered}
u_{i t}-u_{(i-1) t}-e_{i t}+a_{i t}=0 \\
a_{i t}+\sum_{j=i}^{\min \left\{i+t_{t}^{o f f},|\mathcal{I}|\right\}} e_{j t} \leq 1 \\
e_{i t}+\sum_{j=i+1}^{\min \left\{i+t_{t}^{o n},|\mathcal{I}|\right\}} a_{j t} \leq 1 \\
u_{i t}, a_{i t}, e_{i t} \in\{0,1\} \cap \mathcal{U}^{T}
\end{gathered}
$$

where (2a) and (2b) define the auxiliary binary variables $a_{i t}$ and $e_{i t}$ to be $a_{i t}=1$ iff $u_{(i-1) t}=1$ and $u_{i t}=0$, and $e_{i t}=1$ iff $u_{(i-1) t}=0$ and $u_{i t}=1$. Subsequently, the minimum in service $\left(t_{t}^{o n}\right)$ and idle $\left(t_{t}^{o f f}\right)$ times are guaranteed by (2b) and (2c), respectively. $\mathcal{U}^{T}$ represents the value of the variables $u_{i t}$, $a_{i t}$ and $e_{i t}$ set by the initial state of the thermal units at the beginning of day $\mathrm{D}$. An alternative formulation of the thermal unit commitment constraints can be found in [21], where only variable $u_{i t}$ is used at the expense of increasing the number of constraints by more than twice.

\section{Optimal Thermal Bidding Model}

In the MIBEL, a simple day-ahead sale bid consists of a stepwise nondecreasing curve defined with up to 25 price-en- ergy blocks. Similar to [12], this stepwise sale bid will be approximated in our model through the optimal thermal bid function $\lambda_{i t}^{*}\left(p_{i t}\right)$, which is a discontinuous linear piecewise nondecreasing function that gives the value of the optimal bid price $\lambda_{i t}^{*}$ at which the thermal generation $p_{i t}$ would be bid at the $i$ th day-ahead auction. It can be shown that under the price-taker assumption and the MIBEL bid rules, the optimal thermal bid function, i.e., the bid function that maximizes the day-ahead benefit function for any given value $b_{i t}^{T}$, regardless of the value of the clearing price, can be expressed as [22] (3) at the bottom of the page, where $[a-b]^{+}=\max \{0, a-b\}, \underline{p}$ and $\bar{p}_{t}$ are the minimum and maximum generation capacity of the thermal unit $t$, respectively. The variable $b_{i t}^{T}$, which is the total energy production of unit $t$ assigned to the whole portfolio of $\mathrm{BCs}$, is defined as

$$
b_{i t}^{T}=\sum_{j \in \mathcal{B}} b_{i t j}^{T} \quad \forall t \in \mathcal{T}, \quad \forall i \in \mathcal{I} .
$$

Equation (3) can be interpreted with the help of Fig. 5 that represents the optimal thermal bid function (thick line) corresponding to four representative values of the BCs energy $b_{i t}^{T}$.

- Case a): This is the case when $b_{i t}^{T}=0$ (the committed thermal unit $t$ doesn't contribute to the BC covering) which coincides with the classical self-commitment problem treated by several authors [11], [12]. In this case, it is well known that the optimal bid strategy for a price-taking GenCo is to bid at the true marginal cost of the unit. By assuming a quadratic thermal generation cost $C^{T}\left(p_{i t}\right)=c_{t}^{b}+c_{t}^{l} p_{i t}+c_{t}^{q}\left(p_{i t}\right)^{2}$, the optimal bidding policy consists of an instrumental $\left(\lambda_{i t}^{*}=0\right)$ sale bid up to the operational minimum limit $\underline{p}_{t}$ to guarantee their acceptance, and the rest of the plant capacity at the marginal price $2 c_{t}^{q} p_{i t}+c_{t}^{l}$, which is the slope of the cost function $C^{T}\left(p_{i t}\right)$. If this sale bid is submitted to the pool, then the bilateral-free day-ahead matched energy under scenario $s$, $p_{i t}^{D, s}$ will be [see Fig. 5(a)]

$$
p_{i t}^{D, s}=\left\{\begin{array}{lll}
\underline{p}_{t}, & \text { if } p_{i t}^{*, s} \leq \underline{p}_{t} & \forall t \in \mathcal{T} \\
\bar{p}_{t}, & \text { if } p_{i t}^{*, s} \geq \bar{p}_{t} & \forall i \in \mathcal{I} \\
p_{i t}^{*, s}, & \text { otherwise } & \forall s \in \mathcal{S}
\end{array}\right.
$$

where $p_{i t}^{*, s}=\left(\lambda_{i}^{D, s}-c_{t}^{l}\right) / 2 c_{t}^{q}$ is the unconstrained maximum of the benefit function

$$
B^{T, s}\left(p_{i t}\right)=\lambda_{i}^{D, s} p_{i t}-C^{T}\left(p_{i t}\right)
$$

for a given thermal unit $t$, period $i$ and scenario $s$. It must be noted that $p_{i t}^{D, s}$ are constant parameters of the model.

$$
\lambda_{i t}^{*}\left(p_{i t}, b_{i t}^{T}\right)=\left\{\begin{array}{ll}
0, & \text { if } 0 \leq p_{i t} \leq\left[\underline{p}_{t}-b_{i t}^{T}\right]^{+} \\
2 c_{t}^{q}\left(p_{i t}+b_{i t}^{T}\right)+c_{t}^{l}, & \text { if }\left[\underline{p}_{t}-b_{i t}^{T}\right]^{+}<p_{i t} \leq \bar{p}_{t}-b_{i t}^{T}
\end{array} \quad \forall i \in \mathcal{I}, \forall t \in \mathcal{T}\right.
$$



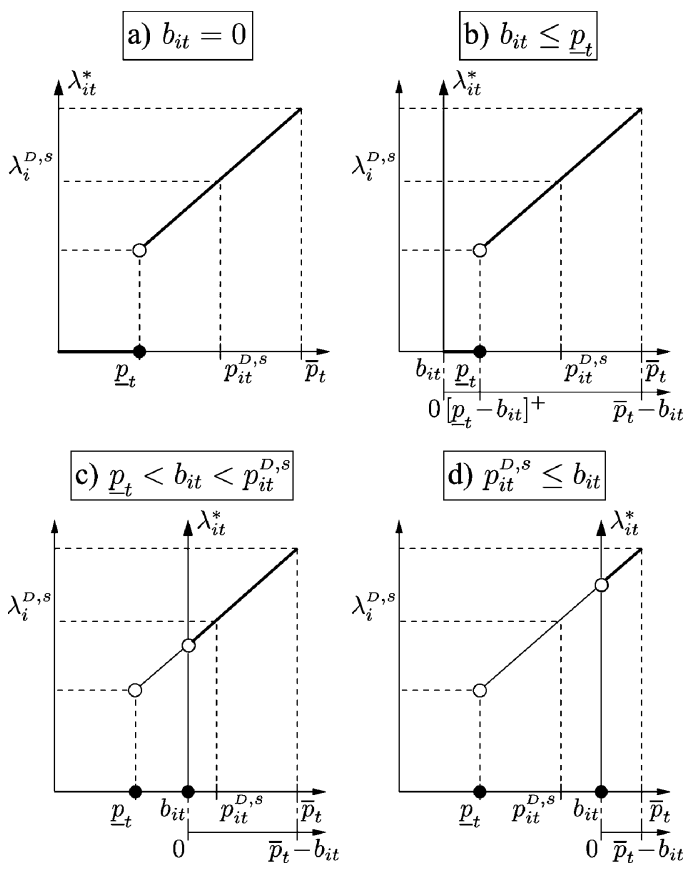

Fig. 5. Representation of the optimal thermal bid function $\lambda_{i t}^{*}\left(p_{i t}, b_{i t}^{T}\right)$.

- Cases b) and c): In both the cases, the energy $b_{i t}^{T}$ to be allocated to the BCs is below the bilateral-free day-ahead matched energy $p_{i t}^{D, s}$ but strictly positive. The MIBEL rules exclude this allocated energy $b_{i t}^{T}$ from the sale bid of the thermal unit, giving rise to the optimal bid curve associated with the second coordinate system of Fig. 5(b) and (c) (thick line), starting at $b_{i t}^{T}$, a value of the original $\mathrm{x}$-axis. In both the cases, the matched energy will be the difference $p_{i t}^{D, s}-b_{i t}^{T}$.

- Cases d): In this last case the allocated energy $b_{i t}^{T}$ exceeds the quantity $p_{i t}^{D, s}$. By looking at the optimal bid curve, it can be observed that the minimum price asked from the market $\lambda_{i t}^{*}\left(0, b_{i t}^{T}\right)=2 c_{t}^{q} b_{i t}^{T}+c_{t}^{l}$ is greater than the represented spot price $\lambda_{i}^{D, s}$ and consequently, the sale bid will remain unmatched.

The thermal matched energy function under scenario $s p_{i t}^{T, s}$ associated with the optimal thermal bidding function (3) (also called price-power function in [14]) was derived in [22], and corresponds to the expression

$$
p_{i t}^{T, s}\left(b_{i t}^{T}, u_{i t}\right)=\left\{\begin{array}{lll}
{\left[p_{i t}^{D, s}-b_{i t}^{T}\right]^{+},} & \text {if } u_{i t}=1 & \forall i \in \mathcal{I} \\
0, & \text { if } u_{i t}=0 & \forall t \in \mathcal{T} \\
& \forall s \in \mathcal{S} .
\end{array}\right.
$$

This discontinuous and nondifferentiable function can be alternatively formulated as a system of linear constraints, called the equivalent thermal-matched energy constraints, with a feasible region represented by the polyhedron $\mathrm{K}_{i t}^{T, s}$ (see Appendix A)

$$
\left[p_{i t}^{T, s}, b_{i t}^{T}, u_{i t}, v_{i t}^{s}, z_{i t}^{s}\right] \in \mathrm{K}_{i t}^{T, s} \quad \forall i \in \mathcal{I}, \quad \forall t \in \mathcal{T}, \quad \forall s \in \mathcal{S}
$$

where $v_{i t}^{s}$ and $z_{i t}^{s}$ are a set of continuous and binary auxiliary variables, respectively. Finally, we can define the second-stage variables $p_{i t}^{s}$ that represent the total generation of the thermal unit $t$ at period $i$ conditioned to scenario $s$, expressed as

$$
\left.\begin{array}{r}
p_{i t}^{s}=p_{i t}^{T, s}+b_{i t}^{T} \\
\underline{p}_{t} u_{i t} \leq p_{i t}^{s} \leq \bar{p}_{t} u_{i t}
\end{array}\right\} \quad \forall i \in \mathcal{I}, \quad \forall t \in \mathcal{T}, \quad \forall s \in \mathcal{S} .
$$

\section{Optimal Generic Programming Unit Bidding Model}

In this section, the optimal bidding and the matched energy functions for a GPU will be derived. First, variable $b_{i}^{G}$ represents the total contribution of the GPU to the coverage of the BCs before the day-ahead market:

$$
b_{i}^{G}=\sum_{j \in \mathcal{B}} b_{i j}^{G} \quad \forall i \in \mathcal{I}
$$

Second, we assume that $p_{i}^{V}$, the exercised energy of the VPP, depends on the value of the binary variable $x_{i}^{V}$ as follows:

$$
p_{i}^{V}=\bar{p}^{V} x_{i}^{V} \quad \forall i \in \mathcal{I}
$$

Under this assumption, the expression of the optimal GPU bid function can be developed by analyzing the two cases, $x_{i}^{V}=0$ and $x_{i}^{V}=1$ :

- $x_{i}^{V}=0$ : VPP rights are not exercised and the energy $b_{i}^{G}$ must be either acquired from the pool or provided by the BCs after the day-ahead market at an agreed price $\lambda^{P}$, which is the maximum price that we were willing to pay to the pool for that amount of energy. Therefore, the optimal purchase bid (energy, price) pair is

$$
\left(b_{i}^{G}, \lambda^{P}\right), \quad \text { if } \quad x_{i}^{V}=0 .
$$

- $x_{i}^{V}=1$ : the VPP rights are exercised and the exercise price is paid. Subsequently, two different situations must be considered:

$-b_{i}^{G} \leq \bar{p}_{i}^{V}$ : After covering the energy $b_{i}^{G}$ with the VPP, there is an energy surplus of $\left[\bar{p}_{i}^{V}-b_{i}^{G}\right]$ that can be sold either to the pool, at unknown spot price $\lambda_{i}^{D}$, or to the BCs after the day-ahead market, at known sale price $\lambda^{S}$. Subsequently, the energy surplus should be offered to the pool at a price not less than $\lambda^{S}$, which is the optimal sale bid

$\left(\left[\bar{p}_{i}^{V}-b_{i}^{G}\right], \lambda^{S}\right), \quad$ if $\quad x_{i}^{V}=1 \quad$ and $\quad b_{i}^{G} \leq \bar{p}_{i}^{V}$.

$-b_{i}^{G}>\bar{p}_{i}^{V}$ : analogously to the case $x_{i}^{V}=0$, to fulfill the uncovered part of the BCs duty, the following optimal purchase bid must be submitted:

$$
\left(\left[b_{i}^{G}-\bar{p}_{i}^{V}\right], \lambda^{P}\right), \quad \text { if } \quad x_{i}^{V}=1 \quad \text { and } \quad b_{i}^{G}>\bar{p}_{i}^{V} .
$$


As a result of the preceding analysis, the optimal sale and purchase bid for the GPU (12)-(14) can be expressed in the following compact form:

$$
\begin{aligned}
\mathrm{OSB}_{i} & =\left(\left[p_{i}^{V}-b_{i}^{G}\right]^{+}, \lambda^{S}\right) \\
\mathrm{OPB}_{i} & =\left(\left[b_{i}^{G}-\bar{p}^{V}\right]^{+}+\min \left\{b_{i}^{G}, \bar{p}^{V}-p_{i}^{V}\right\}, \lambda^{P}\right) .
\end{aligned}
$$

It can be easily verified that for any given value of the first stage variables $b_{i}^{G}$ and $p_{i}^{V}$, (15) and (16) correspond to the optimal bidding rules developed in (12)-(14). Equations (15) and (16) can be used to derive the expressions of the matched energy at each scenario $s \in \mathcal{S}$, as functions of the first stage variables $p_{i}^{V}$ and $b_{i}^{G}$. First, consider the two following sets of scenarios:

$$
\begin{aligned}
& \mathcal{M}_{i}^{S}:=\left\{s \in \mathcal{S} \mid \lambda_{i}^{D, s} \geq \lambda^{S}\right\} \\
& \mathcal{M}_{i}^{P}:=\left\{s \in \mathcal{S} \mid \lambda_{i}^{D, s}<\lambda^{P}\right\}
\end{aligned} \quad \forall i \in \mathcal{I} .
$$

The set $\mathcal{M}_{i}^{S}$ includes those scenarios where, at the $i$ th dayahead auction, the optimal sale bid (14), if any, will be accepted. Then, with respect to (15), the matched sale energy function will be (18) at the bottom of the page. Analogously the set $\mathcal{M}_{i}^{P}$ includes those scenarios where, at the $i$ th day-ahead auction, the optimal purchase bid (16), if any, will be accepted. For the clarity of the exposition, the two terms of the total matched purchase energy of (16) will be represented by two separate matched functions, the matched purchase energy function in (19) at the bottom of the page and the residual matched purchase energy function in (20) at the bottom of the page.

On observing (18), (19), and (20), it becomes evident that actually, the value of the matched sale energy will be the same for any scenario in $\mathcal{M}_{i}^{S}$, and the same happens with the matched purchase energies and the scenarios in $\mathcal{M}_{i}^{P}$. Nevertheless, the superscript " $s$ " will be conserved for the sake of clarity and to strengthen the fact that these are actually second-stage variables, as there will be scenarios with nonzero matched energies, while in others, those energies will be zero. Another issue to mention is that, as we have assumed that $\lambda^{S}<\lambda^{P}$, the intersection set

$$
\mathcal{M}^{S P}:=\mathcal{M}_{i}^{S} \cap \mathcal{M}_{i}^{P}=\left\{s \in \mathcal{S} \mid \lambda_{i}^{D, s} \in\left[\lambda^{S}, \lambda^{P}\right]\right\}
$$

could be nonempty. This fact does not reveal any inconsistency of the model, because (18), (19), and (20) are formulated in a way that, for any $s \in \mathcal{M}^{S P}$, only the matched sale energy $p_{i}^{S, s}$ or the total matched purchase energy $p_{i}^{P, s}+p_{i}^{R, s}$ can be greater than zero, but never simultaneously. Hence, for those scenarios in $\mathcal{M}^{S P}$, only a sale bid or a purchase bid will be submitted, depending on the value of the variables $b_{i}^{G}$ and $p_{i}^{V}$.

Each one of the three nondifferential functions (18), (19), and (20) can be conveniently incorporated into the optimization model through an associated system of equivalent generic matched energy constraints, which is a system of linear constraints with feasible polyhedrons denoted, respectively, by $\mathrm{K}_{i}^{S}$, $\mathrm{K}_{i}^{P}$ and $\mathrm{K}_{i}^{R}$ (see Appendix A):

$$
\left.\begin{array}{cl}
{\left[p_{i}^{S}, p_{i}^{V}, b_{i}^{G}, w_{i}^{S}, y_{i}^{S}\right]} & \in \mathrm{K}_{i}^{S} \\
{\left[p_{i}^{P}, p_{i}^{V}, b_{i}^{G}, w_{i}^{P}, y_{i}^{P}\right]} & \in \mathrm{K}_{i}^{P} \\
{\left[p_{i}^{R}, b_{i}^{G}, w_{i}^{R}, y_{i}^{R}\right]} & \in \mathrm{K}_{i}^{R}
\end{array}\right\} \quad \forall i \in \mathcal{I}
$$

where $w$ and $y$ are a set of continuous and binary auxiliary variables, respectively. The vector $p_{i}^{S}$ are defined as $p_{i}^{S}=\left[p_{i}^{S, 1}, p_{i}^{S, 2}, \ldots, p_{i}^{S,|S|}\right]^{\prime}$, and $p_{i}^{P}$ and $p_{i}^{R}$ are defined analogously.

\section{E. Generic Programming Unit's Net Energy Balance}

At every hour, any GPU operating in the MIBEL must satisfy $i \in \mathcal{I}$, such that the net energy balance of the GPU must be zero, with the help, if necessary, of the BCs after the day-ahead market (see Section III). Following this rule, we assume that, for each scenario $s \in \mathcal{S}$, the energies $b_{i}^{P, s}$ and $b_{i}^{S, s}$ are purchased and sold through these new BCs up to a given maximum quantity at known prices $\lambda^{P}$ and $\lambda^{S}$ (note that $\lambda^{S}<\lambda^{P}$ ), respectively.

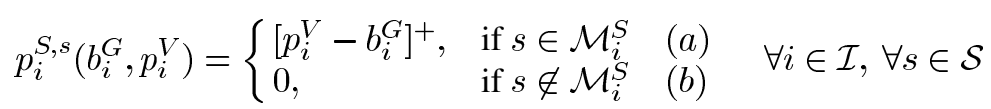

$$
p_{i}^{P, s}\left(b_{i}^{G}, p_{i}^{V}\right)=\left\{\begin{array}{lll}
\min \left\{b_{i}^{G}, \bar{p}^{V}-p_{i}^{V}\right\}, & \text { if } s \in \mathcal{M}_{i}^{P} & (a) \\
0, & \text { if } s \notin \mathcal{M}_{i}^{P} & (b)
\end{array} \quad \forall i \in \mathcal{I}, \forall s \in \mathcal{S}\right.
$$

$$
p_{i}^{R, s}\left(b_{i}^{G}\right)=\left\{\begin{array}{lll}
{\left[b_{i}^{G}-\bar{p}^{V}\right]^{+},} & \text {if } s \in \mathcal{M}_{i}^{P} & (a) \\
0, & \text { if } s \notin \mathcal{M}_{i}^{P} & (b)
\end{array} \quad \forall i \in \mathcal{I}, \forall s \in \mathcal{S}\right.
$$


Thus, the GPU's net energy balance constraints for each hour $i$ and scenario $s$ are

$$
\left.\begin{array}{c}
p_{i}^{V}+p_{i}^{P, s}+p_{i}^{R, s}+b_{i}^{P, s}=p_{i}^{S, s}+b_{i}^{S, s}+b_{i}^{G} \\
0 \leq b_{i}^{P, s} \leq \bar{b}^{P} \\
0 \leq b_{i}^{S, s} \leq \bar{b}^{S}
\end{array}\right\} \forall s \in \mathcal{S}
$$

\section{F. Objective Function}

The expected value of the benefit function $B$ can be expressed as (24)-(28) at the bottom of the page. Equation (24) represents the total income of the BCs before the day-ahead market (constant) and can be ignored in the optimization. However, (25) does not depend on the realization of the random variable $\lambda_{i}^{D, s}$, and corresponds to the on/off fixed cost of the unit commitment and the exercise cost of the VPP energy. Equations (26)-(28) are the expected value of the benefit coming from the day-ahead market's bids of the thermal units (26), the day-ahead market's bids of the GPU (27), and the BCs after the day-ahead market (28), respectively. All the functions in (25)-(28) are linear, except for the generation costs of the thermal units (26), which are concave quadratic $\left(c_{t}^{q} \geq 0\right.$; see Table II).

\section{G. Final Model}

The final model developed in the previous sections is shown in (29) at the bottom of the page with respect to the parameters and sets defined in (5) and (17).

Equation (29) represents the deterministic equivalent program [30] associated with the proposed two-stage stochastic problem with a set $\mathcal{S}$ of scenarios for the spot price $\lambda_{i}^{D}$, where $i \in \mathcal{I}$. The methodology followed to obtain the set $\mathcal{S}$ of scenarios will be presented in Section IV. This deterministic equivalent program corresponds to a mixed continuous-binary linearly constrained maximization problem with concave quadratic objective function. As will be illustrated in Section V, this kind of optimization problems can be solved with the help of standard optimization software, as it is usually carried out in other works on this area (e.g., see [7] and [11]).

\section{Market Price Scenario Generation}

The two-stage stochastic model (29) requires the characterization of the market price through a set of scenarios, also known as a scenario fan. Many scenario generation methods are available (see [26] or [27] for a review on them).

The creation of new BCs and the application of VPP auctions started in June 2007. As the behavior of the prices depends on the market rules, a complete set of 261 equiprobable scenarios

$$
\begin{aligned}
\mathrm{E}_{\lambda^{D}}\left[B\left(u, a, e, p, p^{T}, p^{V}, p^{S}, p^{P}, p^{R}, b^{S}, b^{P} ; \lambda^{D}\right)\right]= & \sum_{i \in \mathcal{I}} \sum_{j \in \mathcal{B}} \lambda_{i j}^{B} L_{i j}^{B} \\
& -\sum_{i \in \mathcal{I}} \sum_{t \in \mathcal{T}}\left[c_{t}^{o n} e_{i t}+c_{t}^{o f f} a_{i t}+c_{t}^{b} u_{i t}\right]-\sum_{i \in \mathcal{I}} \lambda^{V} p_{i}^{V} \\
& +\sum_{i \in \mathcal{I}} \sum_{t \in \mathcal{T}} \sum_{s \in \mathcal{S}} P^{s}\left[\lambda_{i}^{D, s} p_{i t}^{T, s}-c_{t}^{l} p_{i t}^{s}-c_{t}^{q}\left(p_{i t}^{s}\right)^{2}\right] \\
& +\sum_{i \in \mathcal{I}} \sum_{s \in \mathcal{S}} P^{s}\left[\lambda_{i}^{D, s}\left(p_{i}^{S, s}-p_{i}^{P, s}-p_{i}^{R, s}\right)\right] \\
& +\sum_{i \in \mathcal{I}} \sum_{s \in \mathcal{S}} P^{s}\left[\lambda^{S} b_{i}^{S, s}-\lambda^{P} b_{i}^{P, s}\right]
\end{aligned}
$$

$$
\begin{aligned}
& \max \quad \mathrm{E}_{\lambda^{D}}\left[B\left(u, a, e, p, p^{T}, p^{V}, p^{S}, p^{P}, p^{R}, b^{S}, b^{P} ; \lambda^{D}\right)\right] \\
& \text { Eq. (1) Bilateral contracts } \mathcal{B} \text { covering } \\
& \text { Eq. (2) Unit commitment const. } \\
& \text { Eq. (4) Thermal's } b_{i t}^{T} \text { def. } \\
& \text { Eq. (8) Thermal's matched energy } p_{i t}^{T, s} \text { def. } \\
& \text { Eq. (9) Thermal's total generation } p_{i t}^{s} \text { def. } \\
& \text { Eq. (10) GPU's } b_{i}^{G} \text { def. } \\
& \text { Eq. (11) VPP's energy nomination } p_{i}^{V} \text { def. } \\
& \text { Eq. (22) GPU's matched energy } p_{i}^{S / P / R, s} \text { def. } \\
& \text { Eq. (23) GPU's net energy balance const. }
\end{aligned}
$$




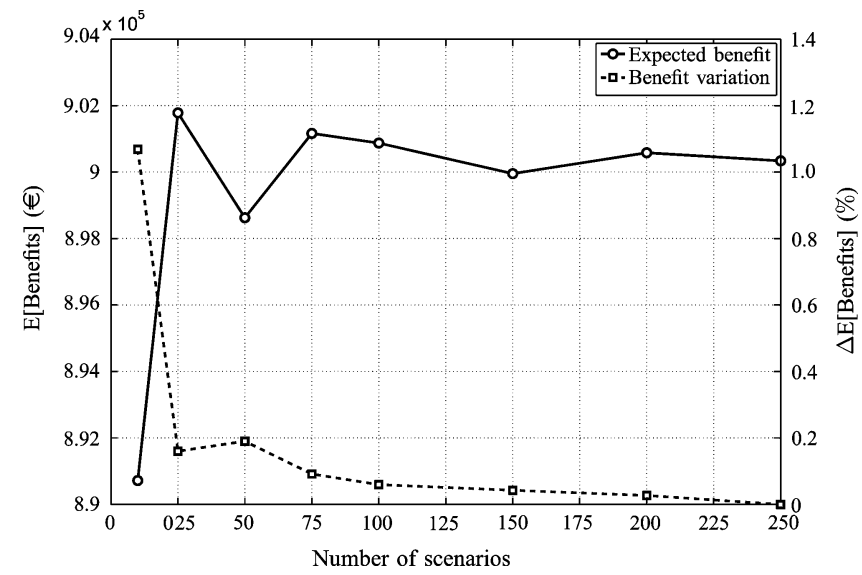

Fig. 6. Expected benefit value and difference between the expected benefit of the complete set and each reduced one, as function of the number of scenarios.

has been obtained using all the available market prices from June 2007 [27].

Given that the size and computational cost of the stochastic programming models depends on the number of scenarios, some scenario reduction techniques have to be applied to reduce the original set of scenarios into a smaller but representative one. We have applied the scenario reduction algorithm explained in [28], which determines a subset of the initial scenario set and assigns new probabilities to the preserved scenarios. For other approaches, see [29].

In our model, a scenario was a set of 24-hourly market prices. The original number of scenarios was 261 . The reduction technique was applied resulting in subsets of $10,25,50,75,100$, 150,200 , and 250 scenarios. Fig. 6 shows how the optimal objective function value changes as the number of scenarios increases. It also contains (right axis) the difference in the percentage between the expected benefits of the complete group of 261 scenarios and each reduced set $(\Delta \mathrm{E}$ [Benefits](\%)). It can be observed that, from 75 scenarios up, any additional increase in the number of scenarios improved the expected benefits by less than $0.09 \%$, while the CPU time increased by almost 14 times (from $442 \mathrm{~s}$ with 75 scenarios to $6554 \mathrm{~s}$ with 100 scenarios). As a consequence, model (29) was tested by a fan with 75 scenarios, for which the objective function value became stable and the computational time cost remained acceptable.

In Table I the stochastic programming indicators needed to evaluate the advantage of the stochastic approximation [30] are reported. EEV is the expected benefit of using the optimal solution of the deterministic problem, where the random variables have been replaced by its mean. RP is the expected benefit of the stochastic problem. Indicator VSS is computed as $\mathrm{VSS}=\mathrm{RP}-\mathrm{EEV}$, and represents a measure of the advantage of using a stochastic programming model instead of a deterministic one. This indicator shows that it is possible to increase the expected benefits by $52.636 €$, by using the stochastic optimal solution. Therefore, we can conclude that the solution obtained through the stochastic programming model increases our expected profits by $6.02 \%$ with respect to the deterministic one.
TABLE I

STOCHASTIC PROGRAMMING INDICATORS

\begin{tabular}{lr}
\hline Monday, April 28, 2008 \\
\hline RP & $901.164 €$ \\
EEV & $848.528 €$ \\
VSS & $52.636 €$ \\
\hline
\end{tabular}

TABLE II

OPERATIONAL CHARACTERISTICS OF THE THERMAL UNITS

\begin{tabular}{|c|c|c|c|c|c|c|c|c|c|c|}
\hline & $\begin{array}{l}c_{t}^{b} \\
€\end{array}$ & $\begin{array}{r}c_{t}^{l} \\
€ / \mathrm{MWh}\end{array}$ & $\begin{array}{r}c_{t}^{q} \\
€ / \mathrm{MWh}^{2}\end{array}$ & $\begin{array}{r}\underline{p}_{t} \\
\mathrm{MW}\end{array}$ & $\begin{array}{r}\bar{p}_{t} \\
\mathrm{MW}\end{array}$ & & $\begin{array}{l}c_{t}^{o n} \\
€\end{array}$ & $\begin{array}{l}c_{t}^{\text {off }} \\
\quad €\end{array}$ & $t^{t} \mathrm{hr}$ & $f^{\prime}$ \\
\hline 1 & 151.08 & 40.37 & 0.015 & 160.0 & 350.0 & +3 & 412.80 & 412.80 & 3 & \\
\hline 2 & 554.21 & & & 250.0 & 563.2 & +3 & & & 3 & \\
\hline 3 & 97.56 & 43.88 & .000 & 80.0 & 284.2 & -3 & 244.80 & 244.80 & 3 & \\
\hline 4 & 327.02 & 28.85 & 0.036 & 160.0 & 370.7 & +3 & 438.40 & 438.40 & 3 & \\
\hline 5 & 64.97 & 5.80 & 0.000 & 30.0 & 65.0 & +3 & 100.20 & 100.20 & 3 & \\
\hline 6 & 366. & -13.7 & .274 & 60.0 & 166.4 & +3 & 188.40 & 188.40 & 3 & \\
\hline 7 & 197.9 & & 0.020 & 160.0 & 364.1 & +3 & 419.20 & 419.20 & 3 & \\
\hline 8 & 66.46 & 55.74 & 0.000 & 110.0 & 313.6 & -3 & 129888 & 1298.88 & 3 & \\
\hline 9 & 66.4 & 5.74 & 0.000 & 110.0 & 313.6 & -3 & 1298.88 & 1298.88 & 3 & 3 \\
\hline & 372.14 & 105.08 & 0.000 & 90.0 & 350.0 & -3 & 1315.44 & 1315.44 & 3 & 3 \\
\hline
\end{tabular}

TABLE III

CHARACTERISTICS OF THE BILATERAL CONTRACTS

\begin{tabular}{ccc}
\hline$j$ & $L_{1 \ldots 24 j}^{B}$ & $\lambda_{1 \ldots 24 j}^{B}$ \\
& $\mathrm{MWh}$ & $€ / \mathrm{MWh}$ \\
\hline 1 & 1100 & 52 \\
2 & 400 & 63 \\
\hline
\end{tabular}

TABLE IV

CHARACTERISTIC OF THE VPP CAPACITY AND THE BC AFTER DAY-AHEAD MARKET

\begin{tabular}{cccccc}
\hline $\bar{p}^{V}$ & $\lambda^{V}$ & $\lambda^{S}$ & $\bar{b}^{S}$ & $\lambda^{B}$ & $\bar{b}^{B}$ \\
MWh & $€ / \mathrm{MWh}$ & $€ / \mathrm{MWh}$ & $\mathrm{MWh}$ & $€ / \mathrm{MWh}$ & $\mathrm{MWh}$ \\
\hline 800 & 38 & 20 & 200 & 100 & 200 \\
\hline
\end{tabular}

\section{Test And Results}

The model (29) has been tested using real data from a Spanish generation company and market prices [23], and the results are reported in this section. The study date was Monday, May 5, 2008. As explained in the earlier sections, a fan with 75 scenarios has been used to represent the spot-price stochasticity. The characteristics of the thermal units, BCs, and VPP capacity are shown in Tables II-IV, respectively. The model (29) has been implemented in AMPL [24] and solved with CPLEX [25] (called with default options) using a SunFire X2200 with two dual core AMD Opteron 2222 processors at $3 \mathrm{GHz}$ and $32 \mathrm{~Gb}$ of RAM memory.

A set of computational tests were performed to evaluate the influence of the GPU and VPP in the GenCo's optimal bidding strategy in the MIBEL. For this reason, the proposed stochastic programming model was tested for three different cases: a) a GenCo with GPU and VPP capacity; b) a GenCo with GPU but without VPP capacity; and c) a GenCo without GPU (see 
TABLE V

OPTIMIZATION CHARACTERISTICS OF THE STUDY CASES

\begin{tabular}{lccccc}
\hline Case & Constraints & $\begin{array}{c}\text { Real } \\
\text { variables }\end{array}$ & $\begin{array}{c}\text { Binary } \\
\text { variables }\end{array}$ & $\begin{array}{c}\text { E(Benefits) } \\
€\end{array}$ & $\begin{array}{c}\text { CPU } \\
\text { s }\end{array}$ \\
\hline (a) & 134034 & 56002 & 18816 & 901.164 & 442 \\
(b) & 128503 & 52364 & 18792 & 665.530 & 214 \\
(c) & 119399 & 46895 & 18720 & 610.264 & 142 \\
\hline
\end{tabular}

Table V for a summary of the optimization problem's dimensions and solutions). The worst expected profit was obtained in case c), where the thermal units were the only ones with the responsibility for fulfilling the $\mathrm{BC}$ before the day-ahead market. Case b) obtained a greater expected profit than case c), owing to the possibility of being able to buy cheaper energy from the pool to cover the $\mathrm{BC}$ and to avoid the use of expensive thermal units. The greatest expected profit was obtained in case a), where the VPP capacity was used to sell in the day-ahead market and to cover part of the BC, using the same advantages of case b).

The optimal management of the GPU in case a) can be analyzed with the help of Figs. 7 and 8. Fig. 7 shows the aggregated economic dispatch of the two BCs (1.500 MWh) by the thermal units $\left(b_{i}^{T}\right.$, white bars) and the GPU ( $b_{i}^{G}$, black bars), together with the exercised VPP energy $p_{i}^{V}$ (small circles). Fig. 8 shows the optimal GPU's sale bid (OSB $i$, positive values) and purchase bid $\left(\mathrm{OPB}_{i}\right.$, negative values) for both cases a) and b) (black and white bars, respectively). On observing both the graphs along the whole 24-h optimization horizon, it is clear that the GPU exhibits a differentiated behavior depending on the time period considered:

- In the time periods $i \in\{5,6,7\}$, the GenCo does not exercise its VPP rights $\left(p_{i}^{V}=0\right)$. For those time periods, all the energy $b_{i}^{G}$ allocated to the BC must be purchased in the day-ahead market (purchase bids, black negative bars in Fig. 8) or from the BC after the day-ahead market. For the rest of the time periods, the GenCo does exercise its VPP rights completely $\left(p_{i}^{V}=\bar{p}_{i}^{V}\right)$.

- There is only one time period $(i=16)$ where the exercised energy coincides with the energy allocated to the $\mathrm{BC}$ $\left(b_{16}^{G}=p_{16}^{V}\right)$.

- For periods $i \in\{3,4,8,21\}$ the allocated energy exceeds the exercised one $\left(b_{i}^{G}>p_{i}^{V}\right)$. The surplus energy, $b_{i}^{G}-p_{i}^{V}$ must be obtained either from the day-ahead market (see the purchase bids for those time periods, black negative bars in Fig. 8) or from the BC after the day-ahead market.

- For periods $i \in\{1,2,9-15,17-20,22-24\}$, only part of the exercised VPP energy is used to satisfy the BC, and the rest is submitted to the day-ahead market (sale bids for those time periods, black positive bars in Fig. 8).

Case b) corresponds to those GenCos operating in the MIBEL, which are not allowed to acquire any VPP capacity rights to prevent these GenCos from becoming price-makers. Under the assumptions of model (29), such a GenCo can use the GPU to purchase energy from the day-ahead market at its best convenience, resulting in an optimal purchase bid pattern that is depicted by the white bars in Fig. 8. The energy of the optimal purchase bid coincides in this case with the contribution of the GPU to the BC at each time period, $b_{i}^{G}$.

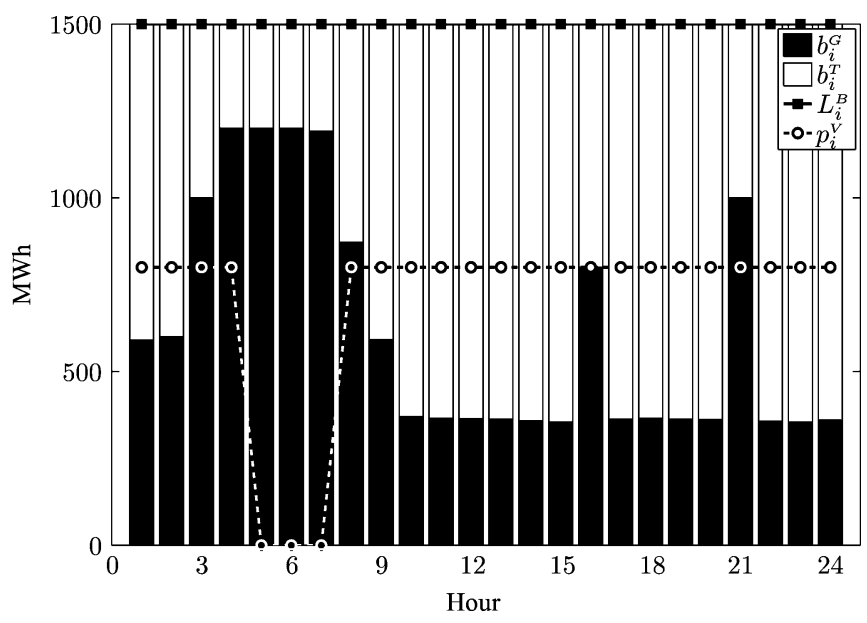

Fig. 7. Aggregated economic dispatch of the two BCs between the thermal units and the GPU for the study case a). Exercised VPP energy is also shown.

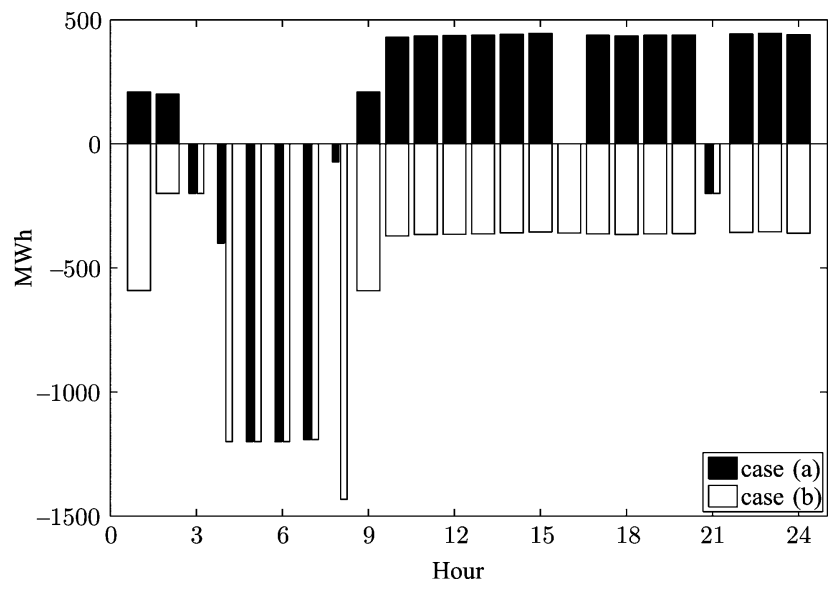

Fig. 8. Sold and bought optimal bidding of the GPU for the study cases a) and b).

Finally, the optimal thermal unit's bidding is analyzed. The thick line in Fig. 9 shows the optimal thermal bid function $\lambda_{i t}^{*}\left(p_{i t}, b_{i t}^{T}\right)$ of the three thermal units $(3,4$, and 6$)$ for all the case studies in each hour. It must be noted that $b_{i}^{T}$ is the energy allocated to the $\mathrm{BC}$ in such a way that the submitted bidding comprises energies between $b_{i}^{T}$ and $\bar{p}_{t}$. The symbol $b_{*}^{T}$ is used to point out the $\mathrm{BC}$ contribution for the remaining hours not shown explicitly in each sub-figure. From Fig. 9, it is clear that the presence of the GPU and VPP capacity allows the thermal units to submit more energy to the pool. For instance, consider the extreme case of thermal unit 3: without GPU [case c)], the generation of this unit is exclusively dedicated to the BC $\left(b_{i, 3}^{T}=\bar{p}_{3} \forall i\right)$, while with GPU and VPP capacity [case a)], all the production output within the operation limits are submitted to the pool $\left(b_{i, 3}^{T}=\underline{p}_{3} \forall i\right)$. The rest of the thermal units exhibit a similar behavior. It can also be observed as to how the availability of the GPU allows the bidding of the thermal unit 6 to adapt itself to the different periods in contrast to case c), where the bidding is almost identical in all the time periods. In general, Fig. 9 shows that the optimal thermal unit's bidding is affected significantly when a GPU is considered, which drastically changes the optimal bidding in a nontrivial 

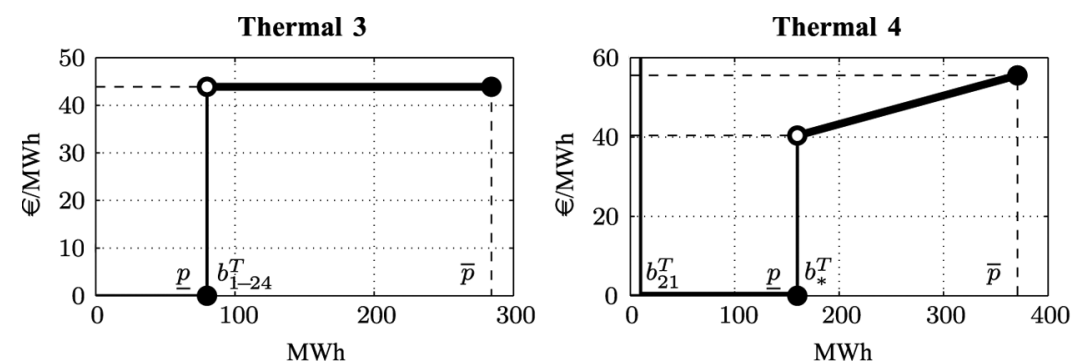

(a)
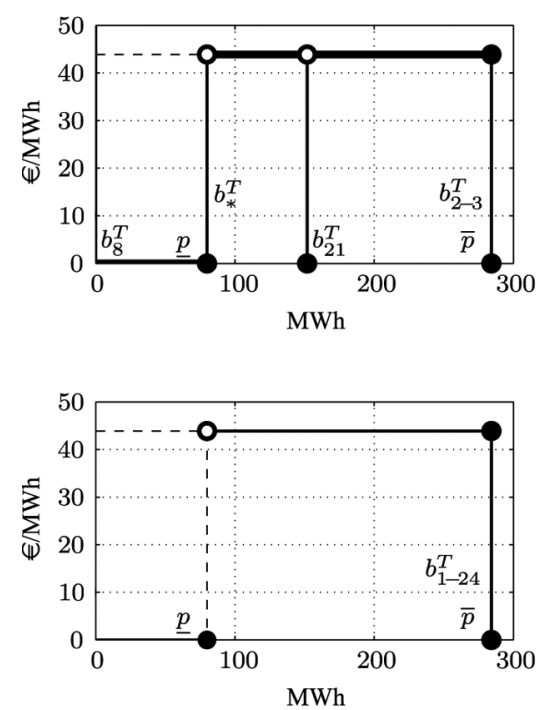

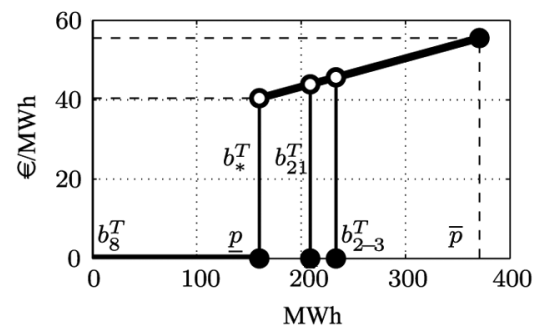

(b)

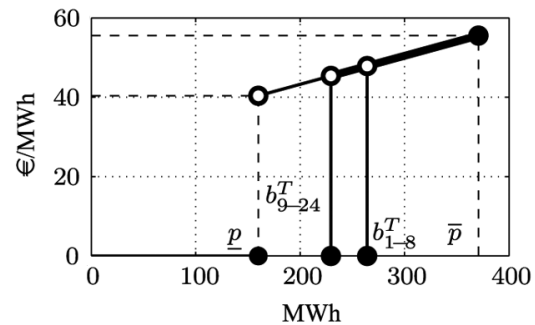

(c)
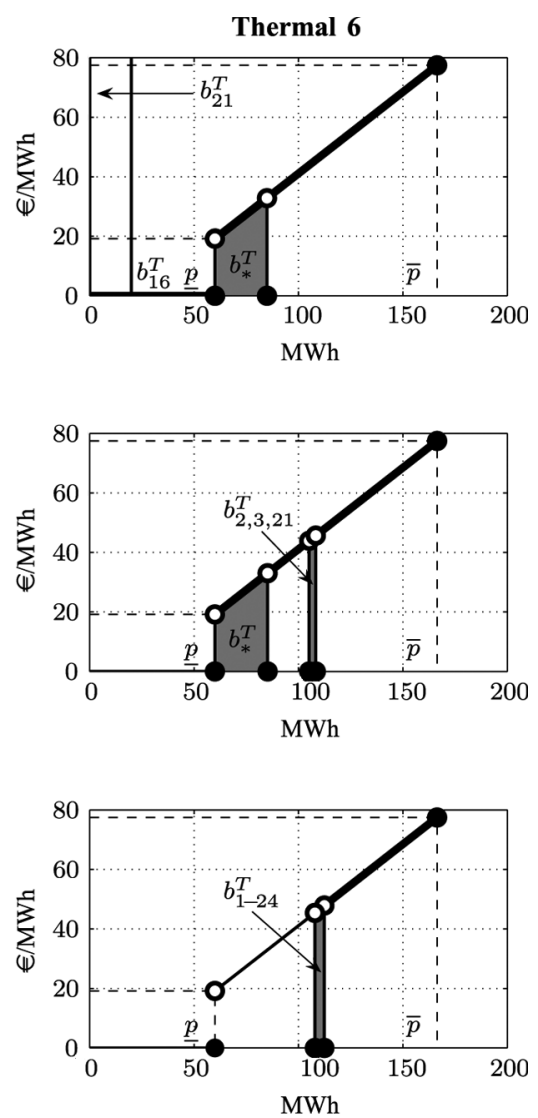

Fig. 9. Bidding curve of thermal production units 3,4 , and 6 for all the case studies. Shadowed zones are regions with a high concentration of values of $b_{i}^{T}$. (a) Case a). (b) Case b). (c) Case c).

way that increases the opportunity of the GenCo to take benefits from the pool.

Finally, we present a comment on the unsuitability of the proposed model to assess the optimal bid of the GenCo in the VPP auction, based on an estimation of the additional profit expectation from holding the VPP. It must be noticed that although a GenCo can use the proposed model to evaluate the expected increase in the profit over a 1-day period, through the direct comparison between the expected benefits of the study cases a) and b) in Table V, the products auctioned in the VPP market are energy delivered over six months and one year. In other words, in order to evaluate the overall expected increase in the benefits, it would be necessary to use mid-term optimization models and not a short-term optimization model such as the one presented in the paper.

\section{CONCLUSIONS}

This paper provides a procedure for a price-taking generation company operating under the most recent regulations of the MIBEL Iberian Electricity Market, to optimally manage a pool of thermal production units and a GPU. The proposed technique has been developed within the versatile decision framework provided by the stochastic programming methodology. A two-stage stochastic mixed quadratic programming problem has been proposed to decide on the optimal unit commitment of the thermal units, the optimal economic dispatch of the BCs between the thermal production units and a GPU and the optimal bid for thermal production units and a GPU observing the MIBEL regulation. The objective of the producers is to maximize the expected profit from their involvement in the spot market, BCs and VPP capacity. The set of scenarios representing the uncertainty of the spot prices are built by applying reduction techniques to the tree obtained from the real data of the MIBEL system. The model was implemented and solved with commercial optimization packages, and tested using the real data of a Spanish generation company and market prices. The results of the computational experiments were reported and analyzed, which demonstrated that the optimal bid policy furnished by the proposed model can increase the day-ahead market benefits of a GenCo operating a GPU at $10 \%$ [case study b) of Table V] or even more, holding a VPP capacity [case study a) of Table V].

\section{APPENDIX \\ EQUiVAlENT MATCHED ENERGY CONSTRAINTS}

In Section III-C, the thermal-matched energy function $p_{i t}^{T, s}$ was defined as

$$
p_{i t}^{T, s}\left(b_{i t}^{T}, u_{i t}\right)=\left\{\begin{array}{llr}
{\left[p_{i t}^{D, s}-b_{i t}^{T}\right]^{+},} & \text {if } u_{i t}=1 & \forall i \in \mathcal{I} \\
0, & \text { if } u_{i t}=0 & \forall t \in \mathcal{T} \\
& & \forall s \in \mathcal{S}
\end{array}\right.
$$

Fig. 10 represents the function $p_{i t}^{T, s}\left(b_{i t}^{T}, u_{i t}\right)$ (thick line and dot), for a fixed value of the spot price $\lambda_{i}^{D, s}$. Although function $p_{i t}^{T, s}\left(b_{i t}^{T}, u_{i t}\right)$ is discontinuous and nondifferentiable, it can be 


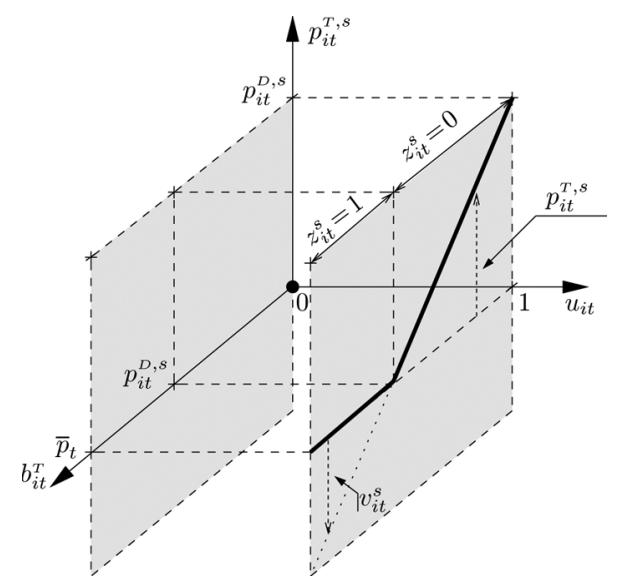

Fig. 10. Thermal matched energy function $p_{i t}^{T, s}$ for a fixed spot price $\lambda_{i}^{D, s}$.

formulated as a system of linear constraints called equivalent thermal-matched energy constraints. With the help of the auxiliary variables $z_{i t}^{s}$ (binary) and $v_{i t}^{s}$ (continuous) (see Fig. 10), the nondifferentiable expression (30) can be shown to be equivalent to the following mixed-integer linear system:

$$
\left.\begin{array}{c}
p_{i t}^{T, s}=p_{i t}^{D, s} u_{i t}+v_{i t}^{s}-b_{i t}^{T} \\
p_{i t}^{D, s}\left(z_{i t}^{s}+u_{i t}-1\right) \leq b_{i t}^{T} \\
b_{i t}^{T} \leq p_{i t}^{D, s}\left(1-z_{i t}^{s}\right)+\bar{p}_{t}\left(z_{i t}^{s}+u_{i t}-1\right) \\
0 \leq p_{i t}^{T, s} \leq p_{i t}^{D, s}\left(1-z_{i t}^{s}\right) \leq p_{i t}^{D, s} u_{i t} \\
0 \leq v_{i t}^{s} \leq\left(\bar{p}_{t}-p_{i t}^{D, s}\right)\left(z_{i t}^{s}+u_{i t}-1\right) \\
b_{i t}^{T} \in\left[0, \bar{p}_{t}\right] \\
z_{i t}^{s} \in\{0,1\}
\end{array}\right\} \forall i \in \mathcal{I}
$$

with a feasible polyhedron denoted by $\mathrm{K}_{i t}^{T, s}$. The equivalence between (30) and (31) indicates that, for every possible value of $u_{i t}$ and $b_{i t}^{T}$, there is a unique feasible value of the matched energy $p_{i t}^{T, s}$ with respect to (31), and that this value satisfies (30). This result has been demonstrated in [22].

In a similar fashion, in Section III-D, the matched sale energy function $p_{i}^{S, s}$, matched purchase energy function $p_{i}^{P, s}$ and

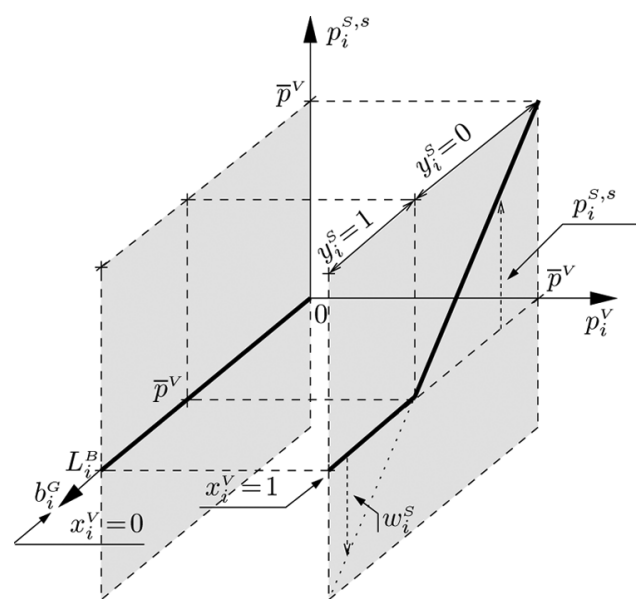

Fig. 11. GPU-matched sale energy function (32a) for $s \in \mathcal{M}_{i}^{S}$.

residual matched purchase energy function $p_{i}^{R, s}$ associated with the GPU were defined as (32)-(34) at the bottom of the page.

These three nondifferential functions (32), (33), (34) can be conveniently incorporated into the optimization model through an equivalent mixed-linear modeling. First, consider (32), which expresses the matched sale energy $p_{i}^{S, s}$ as a function of variables $b_{i}^{G}$ and $p_{i}^{V}$ (see Fig. 11 for a graphical representation of this function). This nondifferential expression can be included into the optimization model through the equivalent set of linear constraints, using the auxiliary variables $w_{i}^{S}$ (continuous) and $y_{i}^{S}$ (binary) shown in (35) at the bottom of the page, and where the constant parameter

$$
L_{i}^{B}=\sum_{\forall j \in \mathcal{B}} L_{i j}^{B} \quad \forall i \in \mathcal{I}
$$

that represents the total energy of the BCs at interval $i$, is used as a trivial upper bound of the variable $b_{i}^{G}$. We denote the feasible polyhedron of (35) by $\mathrm{K}_{i}^{S}$. The equivalence between (32) and (35) can be easily observed, in the sense that for every possible combination of the values of variables $b_{i}^{G}$ and $p_{i}^{V}$, the value uniquely assigned by (35) to the matched sale energy variable $p_{i t}^{S, s}$ satisfies (32). First, it can be observed that for those

$$
\begin{aligned}
p_{i}^{S, s}\left(b_{i}^{G}, p_{i}^{V}\right) & =\left\{\begin{array}{lll}
{\left[p_{i}^{V}-b_{i}^{G}\right]^{+},} & \text {if } s \in \mathcal{M}_{i}^{S} & (a) \\
0, & \text { if } s \notin \mathcal{M}_{i}^{S} & (b)
\end{array} \forall i \in \mathcal{I}, \forall s \in \mathcal{S}\right. \\
p_{i}^{P, s}\left(b_{i}^{G}, p_{i}^{V}\right) & =\left\{\begin{array}{lll}
\min \left\{b_{i}^{G}, \bar{p}^{V}-p_{i}^{V}\right\}, & \text { if } s \in \mathcal{M}_{i}^{P} & (a) \\
0, & \text { if } s \notin \mathcal{M}_{i}^{P} & (b)
\end{array} \forall i \in \mathcal{I}, \forall s \in \mathcal{S}\right. \\
p_{i}^{R, s}\left(b_{i}^{G}\right) & =\left\{\begin{array}{lll}
{\left[b_{i}^{G}-\bar{p}^{V}\right]^{+},} & \text {if } s \in \mathcal{M}_{i}^{P} & (a) \\
0, & \text { if } s \notin \mathcal{M}_{i}^{P} & (b)
\end{array} \forall i \in \mathcal{I}, \forall s \in \mathcal{S}\right.
\end{aligned}
$$

$$
\begin{array}{ccc}
p_{i}^{S, s}=0 & \forall s \notin \mathcal{M}_{i}^{S} & (a) \\
p_{i}^{S, s}=p_{i}^{V}+w_{i}^{S}-b_{i}^{G} & \forall s \in \mathcal{M}_{i}^{S} & (b) \\
0 \leq p_{i}^{S, s} \leq \bar{p}^{V}\left(1-y_{i}^{S}\right) \leq p_{i}^{V} & \forall s \in \mathcal{M}_{i}^{S} & (c) \\
\bar{p}^{V}\left(y_{i}^{S}-1\right)+p_{i}^{V} \leq b_{i}^{G} & & (d) \\
b_{i}^{G} \leq \bar{p}^{V}\left(1-y_{i}^{S}\right)+L_{i}^{B} y_{i}^{S} & & (e) \\
0 \leq w_{i}^{S} \leq\left(L_{i}^{B}-\bar{p}^{V}\right) y_{i}^{S}+\bar{p}^{V}-p_{i}^{V} & & (f) \\
y_{i}^{S} \in\{0,1\} &
\end{array} \quad \forall i \in \mathcal{I}
$$




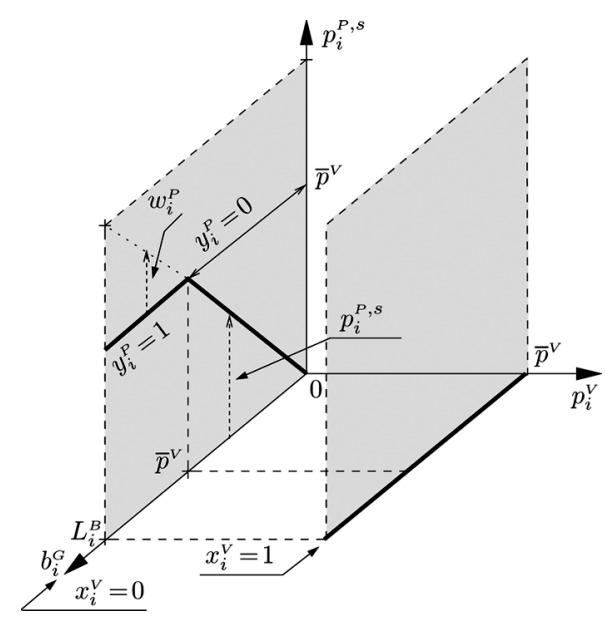

Fig. 12. GPU-matched purchase energy function (33a) for $s \in \mathcal{M}_{i}^{P}$.

$s \notin \mathcal{M}_{i}^{S}$ both (32) and (35) zeroes $p_{i t}^{S, s}$. We analyzed the equivalency for the remaining scenarios $s \in \mathcal{M}_{i}^{S}$ :

- When the nominated VPP energy is $p_{i}^{V}=0$, (35c) sets $y_{i}^{s}=1$ and $p_{i}^{S, s}=0$, which coincides with the value of the matched sale energy function associated with (32). The rest of the system (35) is reduced to the redundant condition $0 \leq w_{i}^{s}=b_{i}^{G} \leq L_{i}^{B}$.

- In case $p_{i}^{V}=\bar{p}^{V}$, if $b_{i}^{G} \leq p_{i}^{V}$, the value of the matched sale energy function (32) will be $p_{i}^{S, s}\left(b_{i}^{G}, \bar{p}^{V}\right)=p_{i}^{V}-b_{i}^{G}$. It is easy to verify that when $b_{i}^{G} \leq p_{i}^{V}$, (35d) and (35f) sets $y_{i}^{S}=0$ and $w_{i}^{S}=0$, and consequently, by (35b), $p_{i t}^{S, s}=p_{i}^{V}-b_{i}^{G}$, which is the same value given by the function (32). The remaining equations of the system (35) provide redundant bounds.

- Finally, when $p_{i}^{V}=\bar{p}^{V}$ and $b_{i}^{G}>p_{i}^{V}$, expression (32) gives $p_{i}^{S, s}\left(b_{i}^{G}, \bar{p}^{V}\right)=0$. By assuming $b_{i}^{G}>p_{i}^{V}$, the only feasible value of $y_{i}^{S}$ permitted by (35e) is $y_{i}^{S}=1$, which, together with (35c), determines $p_{i t}^{S, s}=0$. The rest of the system (35) derives redundant expressions.

By applying a similar analysis to the expression of the matched purchase energy function (33), represented graphically in Fig. 12, it is possible to verify the equivalence between expression (33) and the system of linear constraints (37), with feasible region $\mathrm{K}_{i}^{P}$; see (37) at the bottom of the page, where again, the auxiliary variables $w_{i}^{P}$ (continuous) and $y_{i}^{P}$ (binary) were introduced. Finally, proceeding in a similar way, the

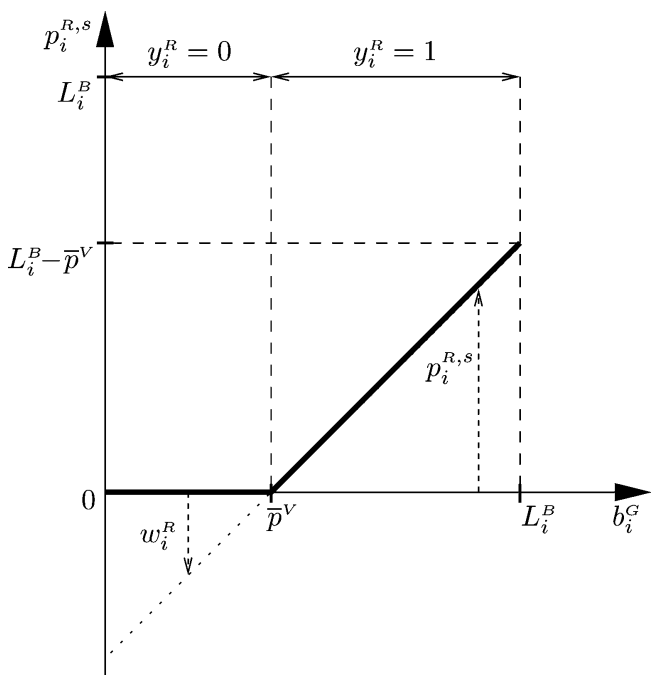

Fig. 13. GPU residual matched purchase energy function (34a) for $s \in \mathcal{M}_{i}^{P}$.

residual matched-purchase energy function (34), represented in Fig. 13, is introduced in the model through the following set of equivalent linear constraints:

$$
\begin{aligned}
& \begin{aligned}
& p_{i}^{R, s}=0 \\
= & b_{i}^{G}+w_{i}^{R}-\bar{p}^{V}
\end{aligned} \\
& \begin{array}{c}
p_{i}^{R, s}=b_{i}^{G}+w_{i}^{R}-\bar{p} \\
0 \leq p_{i}^{R, s} \leq L_{i}^{B} y_{i}^{R}
\end{array} \\
& \bar{p}^{V} y_{i}^{R} \leq b_{i}^{G} \\
& b_{i}^{G} \leq \bar{p}^{V}\left(1-\bar{y}_{i}^{R}\right)+L_{i}^{B} y_{i}^{R} \\
& \begin{array}{c}
0 \leq w_{i}^{R} \leq \bar{p}^{V}\left(1-y_{i}^{R}\right) \\
y_{i}^{R} \in\{0,1\}
\end{array} \\
& \left.\begin{array}{l}
\forall s \notin \mathcal{M}_{i}^{P} \\
\forall s \in \mathcal{M}_{i}^{P} \\
\forall s \in \mathcal{M}_{i}^{P}
\end{array}\right\} \quad \forall i \in \mathcal{I}
\end{aligned}
$$

with a feasible polyhedron denoted by $\mathrm{K}_{i}^{R}$. Again, $w_{i}^{R}$ (continuous) and $y_{i}^{R}$ (binary) represent auxiliary variables.

\section{ACKNOWLEDGMENT}

The author would like to thank Dr. N. Nabona, Dr. J. Castro, Dr. A. Pagès, and Mr. M. Tesser from the Group on Numerical Optimization and Modeling at the Technical University of Catalonia; Mr. A. Canoyra from Union Fenosa; and Ms. T. Sanz and Mr. E. Cortes from Gas Natural, for their valuable comments and suggestions. We appreciate the constructive and fair critique of the four anonymous referees who helped us to improve the original version of the manuscript.

$$
\left.\begin{array}{cc}
p_{i}^{P, s}=0 & \forall s \notin \mathcal{M}_{i}^{P} \\
p_{i}^{P, s}=b_{i}^{G}-w_{i}^{P} & \forall s \in \mathcal{M}_{i}^{P} \\
\bar{p}^{V} y_{i}^{P} \leq p_{i}^{P, s} \leq \bar{p}^{V}-p_{i}^{V} & \forall s \in \mathcal{M}_{i}^{P} \\
\bar{p}^{V} y_{i}^{P} \leq b_{i}^{G} & \\
b_{i}^{G} \leq \bar{p}^{V}\left(1-y_{i}^{P}\right)+L_{i}^{B}\left(y_{i}^{P}+x_{i}^{V}\right)-p_{i}^{V} & \\
0 \leq w_{i}^{P} \leq\left(L_{i}^{B}-\bar{p}^{V}\right) y_{i}^{P}+L_{i}^{B} x_{i}^{V} & \\
y_{i}^{P} \in\{0,1\} &
\end{array}\right\} \quad \forall i \in \mathcal{I}
$$




\section{REFERENCES}

[1] Procedimiento de Operación 3.1 Resolución de 26 de Junio de la Secretaria General de Energía (B.O.E. Núm. 155, de 29 de Junio de 2007), 2007.

[2] Real Decreto de 29 de Diciembre, Disposición Adicional Vigésima Emisiones Primarias de Energia (B.O.E. Núm. 312, de 30 de Diciembre de 2006), 2006.

[3] MIBEL's Virtual Power Plant Auctions, 2009. [Online]. Available: https://www.subasta-epe.com/.

[4] Electricité de France Capacity Auctions, 2008. [Online]. Available: http://capacityauctions.edf.fr/.

[5] Belpex's Virtual Power Plant Auctions, 2008. [Online]. Available: http://www.belpexvpp.be/.

[6] E.ON Energy Sales Virtual Power Plant Auctions, 2008. [Online]. Available: http://www.eon-energy-sales.com/.

[7] M. A. Plazas, A. J. Conejo, and F. J. Prieto, "Multimarket optimal bidding for a power producer," IEEE Trans. Power Syst., vol. 20, no. 4, pp. 2041-2050, Nov. 2005.

[8] E. J. Anderson and A. B. Philpott, "Optimal offer construction in electricity markets," Math. Oper. Res., vol. 27, no. 1, pp. 82-100, 2002.

[9] E. J. Anderson and A. B. Philpott, "Using supply functions for offering market generation into an electricity market," Oper. Res., vol. 50, no. 3 , pp. 477-489, 2003.

[10] P. Neame, A. B. Philpott, and G. Pritchard, "Offer stack optimisation in electricity pool markets," Oper. Res., vol. 51, no. 3, pp. 397-408, 2003.

[11] A. J. Conejo, F. J. Nogales, and J. M. Arroyo, "Price-taker bidding strategy under price uncertainty," IEEE Trans. Power Syst., vol. 17, no. 4, pp. 1081-1088, Nov. 2002.

[12] V. P. Gountis and A. G. Bakirtzis, "Bidding strategies for electricity producers in a competitive electricity marketplace," IEEE Trans. Power Syst., vol. 19, no. 1, pp. 356-365, Feb. 2004.

[13] C. Corchero and F. J. Heredia, "A stochastic programming model for the thermal optimal day-ahead bid problem with physical futures contracts," E-prints UPC. [Online]. Available: http://hdl.handle.net/2117/ 2795.

[14] E. Ni, P. B. Luh, and S. Rourke, "Optimal integrated generation bidding and scheduling with risk management under a deregulated power market," IEEE Trans. Power Syst., vol. 19, no. 1, pp. 600-609, Feb. 2004.

[15] M. Nowak, R. Schultz, and M. Westphalen, "A stochastic integer programming model for incorporating day-ahead trading of electricity into hydro-thermal unit commitment," Optim. Eng., vol. 6, pp. 163-176, 2005.

[16] G. B. Shrestha, S. Kai, and L. Goel, "An efficient stochastic self-scheduling technique for power producers in the deregulated power market," Elect. Power Syst. Res., vol. 71, pp. 91-98, 2004.

[17] C. Triki, P. Beraldi, and G. Gross, "Optimal capacity allocation in multi-auction electricity markets under uncertainty," Comput. Oper. Res., vol. 32, no. 2, pp. 201-217, 2005.

[18] A. J. Conejo, R. Garcia-Bertrand, M. Carrion, A. Caballero, and A. de Andres, "Optimal involvement in futures markets of a power producer," IEEE Trans. Power Syst., vol. 23, no. 2, pp. 703-711, May 2008.

[19] Auction of Bilateral Contracts for Regulated Supply, 2008. [Online]. Available: http://www.subastascesur.omel.es/.

[20] N. Nabona and A. Pages, "A three-stage short-term electric power planning procedure for a generation company in a liberalized market," Int. J. Elect. Power Syst., vol. 29, no. 5, pp. 408-421, 2007.
[21] M. Carrión and J. M. Arroyo, "A computationally efficient mixed-integer linear formulation for the thermal unit commitment problem," IEEE Trans. Power Syst., vol. 21, no. 3, pp. 1371-1378, Aug. 2006.

[22] F. J. Heredia, M. J. Rider, and C. Corchero, "A stochastic programming model for the optimal electricity market bid problem with bilateral contracts for thermal and combined cycle units," E-prints UPC. [Online]. Available: http://hdl.handle.net/2117/2282.

[23] Market Operator of the Electricity Market of Mainland Spain. OMEL, 2008. [Online]. Available: http://www.omel.es/.

[24] R. Fourer, D. M. Gay, and B. W. Kernighan, AMPL: A Modeling Language for Mathematical Programming, 2nd ed. Pacific Grove, CA: Brooks/Cole-Thomson Learning, 2003.

[25] CPLEX Optimization Subroutine Library Guide and Reference, Version 11.0. Incline Village, NV: CPLEX Division, ILOG, Inc., 2008.

[26] M. Kaut and S. W. Wallace, "Evaluation of scenario-generation methods for stochastic programming," SPEPS, Working Paper, vol. 14, 2003.

[27] J. Dupacová, G. Consigli, and S. W. Wallace, "Scenarios for multistage stochastic programs," Ann. Oper. Res., vol. 100, pp. 25-53, 2000.

[28] N. Gröwe-Kuska, H. Heitsch, and W. Römisch, "Scenario reduction and scenario tree construction for power management problems," in Proc. IEEE PowerTech, Bologna, Italy, 2003, vol. 3.

[29] J. M. Morales, S. Pineda, A. J. Conejo, and M. Carrion, "Scenario reduction for futures market trading in electricity markets," IEEE Trans. Power Syst., vol. 24, no. 2, pp. 878-888, May 2009.

[30] J. R. Birge and F. Louveaux, Introduction to Stochastic Programming. New York: Springer-Verlag, 1997.

F. Javier Heredia received the B.Sc. degree in physics from the Universitat de Barcelona, Barcelona, Spain, in 1988 and the Ph.D. degree in operations research from the Universitat Politècnica de Catalunya (UPC), Barcelona, in 1995.

He is currently a Professor of optimization and operations research at the Department of Statistics and Operations Research of the UPC. His current research interests include modeling and optimization of power systems and electricity markets as well as optimization methods such as stochastic and nonlinear programming, dual methods, and networks flows.

Marcos J. Rider (S'97-M'06) received the B.Sc. (Hons.) and P.E. degrees from the National University of Engineering, Lima, Perú, in 1999 and 2000, respectively, the M.Sc. degree from the Federal University of Maranhão, Maranhão, Brazil, in 2002, and the Ph.D. degree from University of Campinas, Campinas, Brazil, in 2006, all in electrical engineering.

Currently, he is carrying out post-doctorate work at the Universitat Politècnica de Catalunya, Barcelona, Spain. His areas of research are the development of methodologies for the optimization, planning and control of electrical power systems, electrical power system analysis in competitive environment and applications of artificial intelligence in power systems.

Cristina Corchero received the B.Sc. degree in statistics and operations research from the Universitat Politècnica de Catalunya, Barcelona, Spain, in 2004. She is currently pursuing the Ph.D. degree at the Universitat Politècnica de Catalunya.

Her research interests are in the fields of power system economics, stochastic programming, and electricity markets. 